\title{
CLASSIFICATION OF REDUCING SUBSPACES OF A CLASS OF MULTIPLICATION OPERATORS ON THE BERGMAN SPACE VIA THE HARDY SPACE OF THE BIDISK
}

\author{
SHUNHUA SUN, DECHAO ZHENG AND CHANGYONG ZHONG
}

\begin{abstract}
In this paper we obtain a complete description of nontrivial minimal reducing subspaces of the multiplication operator by a Blaschke product with four zeros on the Bergman space of the unit disk via the Hardy space of the bidisk.
\end{abstract}

Let $\mathbb{D}$ be the open unit disk in $\mathbb{C}$. Let $d A$ denote Lebesgue area measure on the unit disk $\mathbb{D}$, normalized so that the measure of $\mathbb{D}$ equals 1 . The Bergman space $L_{a}^{2}$ is the Hilbert space consisting of the analytic functions on $\mathbb{D}$ that are also in the space $L^{2}(\mathbb{D}, d A)$ of square integrable functions on $\mathbb{D}$. For a bounded analytic function $\phi$ on the unit disk, the multiplication operator $M_{\phi}$ with symbol $\phi$ is defined on the Bergman space $L_{a}^{2}$ given by

$$
M_{\phi} h=\phi h
$$

for $h \in L_{a}^{2}$. On the basis $\left\{e_{n}\right\}_{n=0}^{\infty}$, where $e_{n}$ is equal to $\sqrt{n+1} z^{n}$, the multiplication operator $M_{z}$ by $z$ is a weighted shift operator, said to be the Bergman shift:

$$
M_{z} e_{n}=\sqrt{\frac{n+1}{n+2}} e_{n+1} \text {. }
$$

A reducing subspace $M$ for an operator $T$ on a Hilbert space $H$ is a subspace $M$ of $H$ such that $T M \subset M$ and $T^{*} M \subset M$. A reducing subspace $M$ of $T$ is called minimal if $M$ does not have any nontrivial subspaces which are reducing subspaces. The goal of this paper is to classify reducing subspaces of $M_{\phi}$ for the Blaschke product $\phi$ with four zeros by identifying its minimal reducing subspaces. Our main idea is to lift the Bergman shift up as a compression of a commuting pair of isometries on a nice subspace of the Hardy space of the bidisk. This idea was used in studying the Hilbert modules by R. Douglas and V. Paulsen [5], operator theory in the Hardy space over the bidisk by R. Dougals and R. Yang [6], [18], [19] and [20]; the higher-order Hankel forms by S. Ferguson and R. Rochberg [7] and [8] and and the lattice of the invariant subspaces of the Bergman shift by S. Richter [12].

On the Hardy space of the unit disk, for an inner function $\phi$, the multiplication operator by $\phi$ is a pure isometry. So its reducing subspaces are in one-to-one correspondence with the closed subspaces of $H^{2} \ominus \phi H^{2}$ [4], [10]. Therefore, it has infinitely many reducing subspaces provided that $\phi$ is any inner function other than a Möbius function. Many people have studied the problem of determining reducing subspaces of a multiplication operator on the Hardy space of the unit circle [1], [2] and [11].

The multiplication operators on the Bergman space possess a very rich structure theory. Even the lattice of the invariant subspaces of the Bergman shift $M_{z}$ is huge [3]. But the lattice of reducing subspaces of the multiplication operator by a finite Blaschke on the

The first author was supported in part by the National Natural Science Foundation-10471041 of China. The second author was partially supported by the National Science Foundation. 
Bergman space seems to be simple. On the Bergman space, Zhu [21] showed that for a Blaschke product $\phi$ with two zeros, the multiplication operator $M_{\phi}$ has exact two nontrivial reducing subspaces $\mathcal{M}_{0}$ and $\mathcal{M}_{0}^{\perp}$. In fact, the restriction of the multiplication operator on $\mathcal{M}_{0}$ is unitarily equivalent to the Bergman shift. Using the Hardy space of bidisk in [9], we show that the multiplication operator with a finite Blaschke product $\phi$ has a unique reducing subspace $\mathcal{M}_{0}(\phi)$, on which the restriction of $M_{\phi}$ is unitarily equivalent to the Bergman shift and if a multiplication operator has a such reducing subspace, then its symbol must be a finite Blaschke product. The space $\mathcal{M}_{0}(\phi)$ is called the distinguished reducing subspace of $M_{\phi}$ and is equal to

$$
\bigvee\left\{\phi^{\prime} \phi^{n}: n=0,1, \cdots, m, \cdots\right\}
$$

if $\phi$ vanishes at 0 in [15], i.e,

$$
\phi(z)=c z \prod_{k=1}^{n} \frac{z-\alpha_{k}}{1-\overline{\alpha_{k}} z},
$$

for some points $\left\{\alpha_{k}\right\}$ in the unit disk and a unimodular constant $c$. The space has played an important role in classifying reducing subspaces of $M_{\phi}$. In [9], we have shown that for a Blaschke product $\phi$ of the third order, except for a scalar multiple of the third power of a Möbius transform, $M_{\phi}$ has exactly two nontrivial minimal reducing subspaces $\mathcal{M}_{0}(\phi)$ and $\mathcal{M}_{0}(\phi)^{\perp}$. This paper continues our study on reducing subspaces of the multiplication operators $M_{\phi}$ on the Bergman space in [9] by using the Hardy space of the bidisk. We will obtain a complete description of nontrivial minimal reducing subspaces of $M_{\phi}$ for the fourth order Blaschke product $\phi$.

This paper is organized as follows. In Section 1 we introduce some notation to lift the Bergman shift as the compression of some isometry on a subspace of the Hardy space of the bidisk and state some theorems in [9] which will be used later. In Section 2 we state the main result and present its proof. Since the proof is long, two difficult cases in the proof are considered in the last two sections.

\section{BERGMAN SPACE VIA HARDY SPACE}

Let $\mathbb{T}$ denote the unit circle. The torus $\mathbb{T}^{2}$ is the Cartesian product $\mathbb{T} \times \mathbb{T}$. Let $d \sigma$ be the rotation invariant Lebesgue measure on $\mathbb{T}^{2}$. The Hardy space $H^{2}\left(\mathbb{T}^{2}\right)$ is the subspace of $L^{2}\left(\mathbb{T}^{2}, d \sigma\right)$, where functions in $H^{2}\left(\mathbb{T}^{2}\right)$ can be identified with the boundary value of the function holomorphic in the bidisc $\mathbb{D}^{2}$ with the square summable Fourier coefficients. The Toeplitz operator on $H^{2}\left(\mathbb{T}^{2}\right)$ with symbol $f$ in $L^{\infty}\left(\mathbb{T}^{2}, d \sigma\right)$ is defined by

$$
T_{f}(h)=P(f h),
$$

for $h \in H^{2}\left(\mathbb{T}^{2}\right)$ where $P$ is the orthogonal projection from $L^{2}\left(\mathbb{T}^{2}, d \sigma\right)$ onto $H^{2}\left(\mathbb{T}^{2}\right)$.

For each integer $n \geq 0$, let

$$
p_{n}(z, w)=\sum_{i=0}^{n} z^{i} w^{n-i} .
$$

Let $\mathcal{H}$ be the subspace of $H^{2}\left(\mathbb{T}^{2}\right)$ spanned by functions $\left\{p_{n}\right\}_{n=0}^{\infty}$. Thus

$$
H^{2}\left(\mathbb{T}^{2}\right)=\mathcal{H} \oplus \operatorname{cl}\left\{(z-w) H^{2}\left(\mathbb{T}^{2}\right)\right\} .
$$

Let

$$
\mathcal{B}=\left.P_{\mathcal{H}} T_{z}\right|_{\mathcal{H}}=\left.P_{\mathcal{H}} T_{w}\right|_{\mathcal{H}}
$$


where $P_{\mathcal{H}}$ is the orthogonal projection from $L^{2}\left(\mathbb{T}^{2}, d \sigma\right)$ onto $\mathcal{H}$. So $\mathcal{B}$ is unitarily equivalent to the Bergman shift $M_{z}$ on the Bergman space $L_{a}^{2}$ via the following unitary operator $U$ : $L_{a}^{2}(\mathbb{D}) \rightarrow \mathcal{H}$,

$$
U z^{n}=\frac{p_{n}(z, w)}{n+1} .
$$

This implies that the Bergman shift is lifted up as the compression of an isometry on a nice subspace of $H^{2}\left(\mathbb{T}^{2}\right)$. Indeed, for each finite Blaschke product $\phi(z)$, the multiplication operator $M_{\phi}$ on the Bergman space is unitarily equivalent to $\phi(\mathcal{B})$ on $\mathcal{H}$.

Let $L_{0}$ be $\operatorname{ker} T_{\phi(z)}^{*} \cap \operatorname{ker} T_{\phi(w)}^{*} \cap \mathcal{H}$. In [9], for each $e \in L_{0}$, we construct functions $\left\{d_{e}^{k}\right\}$ and $d_{e}^{0}$ such that for each $l \geq 1$,

$$
p_{l}(\phi(z), \phi(w)) e+\sum_{k=0}^{l-1} p_{k}(\phi(z), \phi(w)) d_{e}^{l-k} \in \mathcal{H}
$$

and

$$
p_{l}(\phi(z), \phi(w)) e+p_{l-1}(\phi(z), \phi(w)) d_{e}^{0} \in \mathcal{H} .
$$

On one hand, we have a precise formula of $d_{e}^{0}$ :

$$
d_{e}^{0}(z, w)=w e(0, w) e_{0}(z, w)-w \phi_{0}(w) e(z, w),
$$

where $e_{0}$ is the function $\frac{\phi(z)-\phi(w)}{z-w}$. On the other hand, $d_{e}^{k}$ is orthogonal to

$$
\operatorname{ker} T_{\phi(z)}^{*} \cap \operatorname{ker} T_{\phi(w)}^{*} \cap \mathcal{H},
$$

and for a reducing subspace $\mathcal{M}$ and $e \in \mathcal{M}$,

$$
p_{l}(\phi(z), \phi(w)) e+\sum_{k=0}^{l-1} p_{k}(\phi(z), \phi(w)) d_{e}^{l-k} \in \mathcal{M} .
$$

Moreover, the relation between $d_{e}^{1}$ and $d_{e}^{0}$ is given by Theorem 1 in [9] as follows:

Theorem 1.1. If $\mathcal{M}$ is a reducing subspace of $\phi(\mathcal{B})$ orthogonal to the distinguished reducing subspace $\mathcal{M}_{0}$, for each $e \in \mathcal{M} \cap L_{0}$, then there is an element $\tilde{e} \in \mathcal{M} \cap L_{0}$ and a number $\lambda$ such that

$$
d_{e}^{1}=d_{e}^{0}+\tilde{e}+\lambda e_{0} .
$$

Since for Blaschke products with smaller order, it is not difficult to calculate $\tilde{e}$ and $\lambda$ precisely, we are able to classify minimal reducing subspaces of a multiplication operator by a Blaschke product of the fourth order. Main ideas in the proof of Theorems 3.1 and 4.1 are that by complicated computations we use (1.2) to derive conditions on zeros of the Blaschke product of the fourth order.

In this paper we often use Theorem 1.1 and Theorems 1 and 25 in [9] stated as follows.

Theorem 1.2. There is a unique reducing subspace $\mathcal{M}_{0}$ for $\phi(\mathcal{B})$ such that $\left.\phi(\mathcal{B})\right|_{\mathcal{M}_{0}}$ is unitarily equivalent to the Bergman shift. In fact,

$$
\mathcal{M}_{0}=\bigvee_{l \geq 0}\left\{p_{l}(\phi(z), \phi(w)) e_{0}\right\}
$$

and $\left\{\frac{p_{l}(\phi(z), \phi(w)) e_{0}}{\sqrt{l+1}\left\|e_{0}\right\|}\right\}_{0}^{\infty}$ form an orthonormal basis of $\mathcal{M}_{0}$. 
We call $\mathcal{M}_{0}$ to be the distinguished reducing subspace for $\phi(\mathcal{B}) . \mathcal{M}_{0}$ is unitarily equivalent to a reducing subspace of $M_{\phi}$ contained in the Bergman space, denoted by $\mathcal{M}_{0}(\phi)$. The space plays an important role in classifying the minimal reducing subspaces of $M_{\phi}$ in Theorem 2.1.

In [9] we showed that for a nontrivial minimal reducing subspace $\Omega$ for $\phi(\mathcal{B})$, either $\Omega$ equals $\mathcal{M}_{0}$ or $\Omega$ is a subspace of $\mathcal{M}_{0}^{\perp}$. The condition in the following theorem is natural.

Theorem 1.3. Suppose that $\Omega, \mathcal{M}$ and $\mathcal{N}$ are three distinct nontrivial minimal reducing subspaces for $\phi(\mathcal{B})$ and

$$
\Omega \subset \mathcal{M} \oplus \mathcal{N} .
$$

If they are contained in $\mathcal{M}_{0}^{\perp}$, then there is a unitary operator $U: \mathcal{M} \rightarrow \mathcal{N}$ such that $U$ commutes with $\phi(\mathcal{B})$ and $\phi(\mathcal{B})^{*}$.

\section{MAIN RESULT}

Let $\phi$ be a Blaschke product with four zeros. In this section we will obtain a complete description of minimal reducing subspaces of the multiplication operator $M_{\phi}$. First observe that the multiplication operator $M_{z^{4}}$ is a weighted shift with multiplicity 4:

$$
M_{z^{4}} e_{n}=\sqrt{\frac{n+1}{n+5}} e_{n+4}
$$

where $e_{n}$ equals $\sqrt{n+1} z^{n}$. By Theorem B [14], $M_{z^{4}}$ has exact four nontrivial minimal reducing subspaces:

for $j=1,2,3,4$.

$$
\mathcal{M}_{j}=\bigvee\left\{z^{n}: n \equiv j \bmod 4\right\}
$$

Before stating the main result of this paper we need some notation. It is not difficult to see that the set of finite Blaschke products forms a semigroup under composition of two functions. For a finite Blaschke product $\phi$ we say that $\phi$ is decomposable if there are two Blaschke products $\psi_{1}$ and $\psi_{2}$ with orders greater than 1 such that

$$
\phi(z)=\psi_{1} \circ \psi_{2}(z) \text {. }
$$

For each $\lambda$ in $\mathbb{D}$, let $\phi_{\lambda}$ denote the Möbius transform:

$$
\phi_{\lambda}(z)=\frac{\lambda-z}{1-\bar{\lambda} z}
$$

Define the operator $U_{\lambda}$ on the Bergman space as follows:

$$
U_{\lambda} f=f \circ \phi_{\lambda} k_{\lambda}
$$

for $f$ in $L_{a}^{2}$ where $k_{\lambda}$ is the normalized reproducing kernel $\frac{\left(1-|\lambda|^{2}\right)}{(1-\lambda z)^{2}}$. Clearly, $U_{\lambda}$ is a selfadjoint unitary operator on the Bergman space. Using the unitary operator $U_{\lambda}$ we have

$$
\mathcal{M}_{0}(\phi)=U_{\lambda} \mathcal{M}_{0}\left(\phi \circ \phi_{\lambda}\right)
$$

where $\lambda$ is a zero of the finite Blaschke product $\phi$. This easily follows from that $\phi \circ \phi_{\lambda}$ vanishes at 0 and

$$
U_{\lambda}^{*} M_{\phi} U_{\lambda}=M_{\phi \circ \phi_{\lambda}} .
$$

We say that two Blaschke products $\phi_{1}$ and $\phi_{2}$ are equivalent if there is a complex number $\lambda$ in $\mathbb{D}$ such that

$$
\phi_{1}=\phi_{\lambda} \circ \phi_{2}
$$


For two equivalent Blaschke products $\phi_{1}$ and $\phi_{2}, M_{\phi_{1}}$ and $M_{\phi_{2}}$ are mutually analytic function calculus of each other and hence share reducing subspaces. The following main result of this paper gives a complete description of minimal reducing subspaces.

Theorem 2.1. Let $\phi$ be a Blaschke product with four zeros. One of the following holds.

(1) If $\phi$ is equivalent to $z^{4}$, i.e., $\phi$ is a scalar multiple of the fourth power $\phi_{c}^{4}$ of the Möbius transform $\phi_{c}$ for some complex number $c$ in the unit disk, $M_{\phi}$ has exact four nontrivial minimal reducing subspaces

$$
\left\{U_{c} \mathcal{M}_{1}, U_{c} \mathcal{M}_{2}, U_{c} \mathcal{M}_{3}, U_{c} \mathcal{M}_{4}\right\} .
$$

(2) If $\phi$ is is decomposable but not equivalent to $z^{4}$, i.e, $\phi=\psi_{1} \circ \psi_{2}$ for two Blaschke products $\psi_{1}$ and $\psi_{2}$ with orders 2 but not both of $\psi_{1}$ and $\psi_{2}$ are a scalar multiple of $z^{2}$, then $M_{\phi}$ has exact three nontrivial minimal reducing subspaces

$$
\left\{\mathcal{M}_{0}(\phi), \mathcal{M}_{0}\left(\psi_{2}\right) \ominus \mathcal{M}_{0}(\phi), \mathcal{M}_{0}\left(\psi_{2}\right)^{\perp}\right\} .
$$

(3) If $\phi$ is not decomposable, then $M_{\phi}$ has exact two nontrivial minimal reducing subspaces

$$
\left\{\mathcal{M}_{0}(\phi), \mathcal{M}_{0}(\phi)^{\perp}\right\} .
$$

To prove the above theorem we need the following two lemmas which tell us when a Blaschke product with order 4 is decomposable.

Lemma 2.2. If a Blaschke product $\phi$ with order four is decomposable, then the numerator of the rational function $\phi(z)-\phi(w)$ has at least three irreducible factors.

Proof. Suppose that $\phi$ is the Blaschke product with order four. Let $f(z, w)$ be the numerator of the rational function $\phi(z)-\phi(w)$. If $\phi$ is decomposable, then $\phi=\psi_{1} \circ \psi_{2}$ for two Blaschke products $\psi_{1}$ and $\psi_{2}$ with order two. Let $g(z, w)$ be the numerator of the rational function $\psi_{1}(z)-\psi_{1}(w)$. Clearly, $z-w$ is a factor of $g(z, w)$. Thus we can write

$$
g(z, w)=(z-w) p(z, w)
$$

for some polynomial $p(z, w)$ of $z$ and $w$ to get

$$
g\left(\psi_{2}(z), \psi_{2}(w)\right)=\left(\psi_{2}(z)-\psi_{2}(w)\right) p\left(\psi_{2}(z), \psi_{2}(w)\right) .
$$

On the other hand, we also have

$$
\psi_{2}(z)-\psi_{2}(w)=\frac{(z-w) p_{2}(z, w)}{q_{2}(z, w)}
$$

for two polynomials $p_{2}(z, w)$ and $q_{2}(z, w)$ which $p_{2}(z, w)$ and $q_{2}(z, w)$ do not have common factor. In fact, $q_{2}(z, w)$ and the numerator of the rational function $p\left(\psi_{2}(z), \psi_{2}(w)\right)$ do not have common factor also. So we obtain

$$
g\left(\psi_{2}(z), \psi_{2}(w)\right)=\frac{(z-w) p_{2}(z, w)}{q_{2}(z, w)} p\left(\psi_{2}(z), \psi_{2}(w)\right) .
$$

Since $f(z, w)$ is the numerator of the rational function $g\left(\psi_{2}(z), \psi_{2}(w)\right)$, this gives that $f(z, w)$ has at least three factors. This completes the proof.

For $\alpha, \beta \in \mathbb{D}$, define

$$
f_{\alpha, \beta}(w, z)=w^{2}(w-\alpha)(w-\beta)(1-\bar{\alpha} z)(1-\bar{\beta} z)-z^{2}(z-\alpha)(z-\beta)(1-\bar{\alpha} w)(1-\bar{\beta} w) .
$$


It is easy to see that $f_{\alpha, \beta}(w, z)$ is the numerator of $z^{2} \phi_{\alpha}(z) \phi_{\beta}(z)-w^{2} \phi_{\alpha}(w) \phi_{\beta}(w)$. The following lemma gives a criteria when the Blaschke product $z^{2} \phi_{\alpha}(z) \phi_{\beta}(z)$ is decomposable.

Lemma 2.3. For $\alpha$ and $\beta$ in $\mathbb{D}$, one of the following holds.

(1) If both $\alpha$ and $\beta$ equal zero, then

$$
f_{\alpha, \beta}(w, z)=(w-z)(w+z)(w-i z)(w+i z) .
$$

(2) If $\alpha$ does not equal either $\beta$ or $-\beta$, then

$$
f_{\alpha, \beta}(w, z)=(w-z) p(w, z)
$$

for some irreducible polynomial $p(w, z)$.

(3) If $\alpha$ equals either $\beta$ or $-\beta$ but it does not equal zero, then

$$
f_{\alpha, \beta}(w, z)=(w-z) p(w, z) q(w, z)
$$

for two irreducible distinct polynomials $p(w, z)$ and $q(w, z)$.

Proof. Clearly, (1) holds.

To prove (2), by the example on page 6 of [13] we may assume that none of $\alpha$ and $\beta$ equals 0 . First observe that $(w-z)$ is a factor of the polynomial $f_{\alpha, \beta}(w, z)$. Taking a long division gives

$$
f_{\alpha, \beta}(w, z)=(w-z) g_{\alpha, \beta}(w, z)
$$

where

$$
\begin{aligned}
g_{\alpha, \beta}(w, z)= & (1-\bar{\alpha} z)(1-\bar{\beta} z) w^{3}+(z-(\alpha+\beta))(1-\bar{\alpha} z)(1-\bar{\beta} z) w^{2} \\
& +(z-\alpha)(z-\beta)(1-(\bar{\alpha}+\bar{\beta}) z) w+z(z-\alpha)(z-\beta) .
\end{aligned}
$$

Next we will show that $g_{\alpha, \beta}(w, z)$ is irreducible. To do this, we assume that $g_{\alpha, \beta}(w, z)$ is reducible to derive a contradiction.

Assuming that $g_{\alpha, \beta}(w, z)$ is reducible, we can factor $g_{\alpha, \beta}(w, z)$ as the product of two polynomials $p(w, z)$ and $q(w, z)$ of $z$ and $w$ with degree of $w$ greater than or equal one. Write

$$
\begin{aligned}
& p(w, z)=a_{1}(z) w+a_{0}(z) \\
& q(w, z)=b_{2}(z) w^{2}+b_{1}(z) w+b_{0}(z)
\end{aligned}
$$

where $a_{j}(z)$ and $b_{j}(z)$ are polynomials of $z$. Since $g_{\alpha, \beta}(w, z)$ equals the product of $p(w, z)$ and $q(w, z)$, taking the product and comparing coefficients of $w^{k}$ give

$$
\begin{aligned}
a_{1}(z) b_{2}(z) & =(1-\bar{\alpha} z)(1-\bar{\beta} z), \\
a_{1}(z) b_{1}(z)+a_{0}(z) b_{2}(z) & =(z-(\alpha+\beta))(1-\bar{\alpha} z)(1-\bar{\beta} z), \\
a_{1}(z) b_{0}(z)+a_{0}(z) b_{1}(z) & =(z-\alpha)(z-\beta)(1-(\bar{\alpha}+\bar{\beta}) z), \\
a_{0}(z) b_{0}(z) & =z(z-\alpha)(z-\beta) .
\end{aligned}
$$

Equation (2.1) gives that either

$$
\begin{aligned}
& a_{1}(z)=(1-\bar{\alpha} z) \text { or } \\
& a_{1}(z)=(1-\bar{\alpha} z)(1-\bar{\beta} z) \text { or } \\
& a_{1}(z)=1 .
\end{aligned}
$$


In the first case that $a_{1}(z)=(1-\bar{\alpha} z),(2.1)$ gives $b_{2}(z)=(1-\bar{\beta} z)$. Thus by Equation (2.2), we have

$$
a_{0}(z)(1-\bar{\beta} z)=(1-\bar{\alpha} z)\left[(z-(\alpha+\beta))(1-\bar{\beta} z)-b_{1}(z)\right],
$$

to get that $(1-\bar{\alpha} z)$ is a factor of $a_{0}(z)$, and hence is also a factor of a factor $z(z-\alpha)(z-\beta)$ by (2.4). This implies that $\alpha$ must equal 0 . It is a contradiction.

In the second case that $a_{1}(z)=(1-\bar{\alpha} z)(1-\bar{\beta} z)$, we have that $b_{2}(z)=1$ to get that either the degree of $b_{1}(z)$ or the degree of $b_{0}(z)$ must be one while the degrees of $b_{1}(z)$ and $b_{0}(z)$ are at most one. So the degree of $a_{0}(z)$ is at most two. Also $a_{0}(z)$ does not equal zero. Equation (2.2) gives

$$
(1-\bar{\alpha} z)(1-\bar{\beta} z) b_{1}(z)+a_{0}(z)=(z-(\alpha+\beta))(1-\bar{\alpha} z)(1-\bar{\beta} z) .
$$

Thus

$$
a_{0}(z)=c_{1}(1-\bar{\alpha} z)(1-\bar{\beta} z)
$$

for some constant $c_{1}$. But Equation (2.4) gives

$$
c_{1}(1-\bar{\alpha} z)(1-\bar{\beta} z) b_{0}(z)=z(z-\alpha)(z-\beta) .
$$

Either $c_{1}=0$ or $(1-\bar{\alpha} z)(1-\bar{\beta} z)$ is a factor of $z(z-\alpha)(z-\beta)$. This is impossible.

In the third case that $a_{1}(z)=1$, then $b_{2}(z)=(1-\bar{\alpha} z)(1-\bar{\beta} z)$. Since the root $w$ of $f_{\alpha, \beta}(w, z)$ is a nonconstant function of $z$, the degree of $a_{0}(z)$ must be one. Thus the degrees of $b_{1}(z)$ and $b_{0}(z)$ are at most two. By Equation (2.2) we have

$$
(1-\bar{\alpha} z)(1-\bar{\beta} z) a_{0}(z)+b_{1}(z)=(z-(\alpha+\beta))(1-\bar{\alpha} z)(1-\bar{\beta} z),
$$

to get

$$
b_{1}(z)=(1-\bar{\alpha} z)(1-\bar{\beta} z)\left[(z-(\alpha+\beta))-a_{0}(z)\right] .
$$

Since the degree of $b_{1}(z)$ is at most two, we have

$$
\begin{aligned}
& a_{0}(z)=(z-(\alpha+\beta))-c_{0} \\
& b_{1}(z)=c_{0}(1-\bar{\alpha} z)(1-\bar{\beta} z) .
\end{aligned}
$$

Equations (2.4) and (2.3) give

$$
\left[(z-(\alpha+\beta))-c_{0}\right] b_{0}(z)=z(z-\alpha)(z-\beta)
$$

and

$$
\begin{aligned}
& b_{1}(z)\left[(z-(\alpha+\beta))-c_{0}\right]+b_{0}(z) \\
= & (z-\alpha)(z-\beta)(1-(\bar{\alpha}+\bar{\beta}) z) .
\end{aligned}
$$

Multiplying the both sides of the last equality by $\left[(z-(\alpha+\beta))-c_{0}\right]$ gives

$$
\begin{aligned}
& b_{1}(z)\left[(z-(\alpha+\beta))-c_{0}\right]^{2}+z(z-\alpha)(z-\beta) \\
= & {\left[(z-(\alpha+\beta))-c_{0}\right](z-\alpha)(z-\beta)(1-(\bar{\alpha}+\bar{\beta}) z) . }
\end{aligned}
$$

This leads to

$$
\begin{aligned}
& c_{0}(1-\bar{\alpha} z)(1-\bar{\beta} z)\left[(z-(\alpha+\beta))-c_{0}\right]^{2}+z(z-\alpha)(z-\beta) \\
= & {\left[(z-(\alpha+\beta))-c_{0}\right](z-\alpha)(z-\beta)(1-(\bar{\alpha}+\bar{\beta}) z) . }
\end{aligned}
$$

If $c_{0} \neq 0$, then the above equality gives that $(z-\alpha)(z-\beta)$ is a factor of $[(z-(\alpha+\beta))-$ $\left.c_{0}\right]^{2}$. This is impossible. 
If $c_{0}=0$, then we have

$$
z(z-\alpha)(z-\beta)=[(z-(\alpha+\beta))](z-\alpha)(z-\beta)(1-(\bar{\alpha}+\bar{\beta}) z) .
$$

to get

$$
\bar{\alpha}+\bar{\beta}=0
$$

and hence $\alpha=-\beta$. It is also a contradiction. This completes the proof that $g_{\alpha, \beta}(w, z)$ is irreducible.

To prove (3), we note that if $\alpha$ equals $\beta$, an easy computation gives

$$
\begin{aligned}
f_{\alpha, \beta}(w, z)= & (w-z)[(1-\bar{\alpha} z) w+(z-\alpha)] \\
& \times[w(w-\alpha)(1-\bar{\alpha} z)+z(z-\alpha)(1-\bar{\alpha} w)] .
\end{aligned}
$$

If $\alpha=-\beta$, we also have

$$
f_{\alpha, \beta}(w, z)=(w-z)(w+z)\left[\left(1-\bar{\alpha}^{2} z^{2}\right) w^{2}+\left(z^{2}-\alpha^{2}\right)\right] .
$$

This completes the proof.

Proof of Theorem 2.1. Assume that $\phi$ is a Blaschke product with the fourth order. By the Bochner Theorem [17], $\phi$ has a critical point $c$ in the unit disk. Let $\lambda=\phi(c)$ be the critical value of $\phi$. Then there are two points $\alpha$ and $\beta$ in the unit disk such that

$$
\phi_{\lambda} \circ \phi \circ \phi_{c}(z)=\eta z^{2} \phi_{\alpha} \phi_{\beta}
$$

where $\eta$ is a unimodule constant. Let $\psi$ be $z^{2} \phi_{\alpha} \phi_{\beta}$. Since $\phi \circ \phi_{c}$ and $\psi$ are mutually analytic function calculus of each other, both $M_{\phi \circ \phi_{c}}$ and $M_{\psi}$ share reducing subspaces.

(1) If $\phi$ is equivalent to $z^{4}$, then $\psi$ must equal a scalar multiple of $z^{4}$. By Theorem $\mathrm{B}$ in [14], $M_{\psi}$ has exact four nontrivial minimal reducing subspaces

$$
\left\{\mathcal{M}_{1}, \mathcal{M}_{2}, \mathcal{M}_{3}, \mathcal{M}_{4}\right\}
$$

where

$$
\mathcal{M}_{j}=\bigvee\left\{z^{n}: n \equiv j \bmod 4\right\}
$$

for $j=1,2,3,4$. The four spaces above are also reducing subspaces for $M_{\phi \circ \phi_{c}}$. Noting

$$
U_{c}^{*} M_{\phi \circ \phi_{c}} U_{c}=M_{\phi}
$$

we have that $M_{\phi}$ has exact four nontrivial minimal reducing subspaces

$$
\left\{U_{c} \mathcal{M}_{1}, U_{c} \mathcal{M}_{2}, U_{c} \mathcal{M}_{3}, U_{c} \mathcal{M}_{4}\right\}
$$

(2) If $\phi$ is decomposable but not equivalent to $z^{4}$, i.e, $\phi=\psi_{1} \circ \psi_{2}$ for two Blaschke products $\psi_{1}$ and $\psi_{2}$ with degrees two and not both $\psi_{1}$ and $\psi_{2}$ are scalar multiples of $z^{2}$, by Lemmas 2.2 and 2.3, then $\alpha$ equals either $\beta$ or $-\beta$ but does not equal 0 . By Theorem 1.2, the restriction of $M_{\psi_{2}}$ on $\mathcal{M}_{0}\left(\psi_{2}\right)$ is unitarily equivalent to the Bergman shift. Thus $\mathcal{M}_{0}\left(\psi_{2}\right)$ is also a reducing subspace of $M_{\phi}$ and the restriction of $M_{\phi}=M_{\psi_{1} \circ \psi_{2}}$ on $M_{0}\left(\psi_{2}\right)$ is unitarily equivalent to $M_{\psi_{1}}$ on the Bergman space. By Theorem 1.2 again, there is a unique reducing subspace $\mathcal{M}_{0}\left(\psi_{1}\right)$ on which the restriction $M_{\psi_{1}}$ is unitarily equivalent to the Bergman shift. Thus there is a subspace of $\mathcal{M}_{0}\left(\psi_{2}\right)$ on which the restriction of $M_{\phi}$ is unitarily equivalent to the Bergman shift. Theorem 1.2 implies that $\mathcal{M}_{0}(\phi)$ is contained in $\mathcal{M}_{0}\left(\psi_{2}\right)$. Therefore $\mathcal{M}_{0}\left(\psi_{2}\right) \ominus \mathcal{M}_{0}(\phi)$ is also a minimal reducing subspace of $M_{\phi}$ and

$$
L_{a}^{2}=\mathcal{M}_{0}(\phi) \oplus\left[\mathcal{M}_{0}\left(\psi_{2}\right) \ominus \mathcal{M}_{0}(\phi)\right] \oplus\left[\mathcal{M}_{0}\left(\psi_{2}\right)\right]^{\perp} .
$$

By Theorems 3.1 in [16], $\left\{\mathcal{M}_{0}(\phi),\left[\mathcal{M}_{0}\left(\psi_{2}\right) \ominus \mathcal{M}_{0}(\phi)\right],\left[\mathcal{M}_{0}\left(\psi_{2}\right)\right]^{\perp}\right\}$ are nontrivial minimal reducing subspaces of $M_{\phi}$. We will show that they are exact nontrivial minimal reducing 
subspaces of $M_{\phi}$. If this is not true, then there is another minimal reducing subspace $\Omega$ of $M_{\phi}$. By Theorem 38 [9], we have

$$
\Omega \subset\left[\mathcal{M}_{0}\left(\psi_{2}\right) \ominus \mathcal{M}_{0}(\phi)\right] \oplus\left[\mathcal{M}_{0}\left(\psi_{2}\right)\right]^{\perp} .
$$

By Theorem 1.3, there is a unitary operator

$$
U:\left[\mathcal{M}_{0}\left(\psi_{2}\right) \ominus \mathcal{M}_{0}(\phi)\right] \rightarrow\left[\mathcal{M}_{0}\left(\psi_{2}\right)\right]^{\perp}
$$

which commutes with both $M_{\phi}$ and $M_{\phi}^{*}$. But

$$
\operatorname{dimker} M_{\phi}^{*} \cap\left[\mathcal{M}_{0}\left(\psi_{2}\right) \ominus \mathcal{M}_{0}(\phi)\right]=1
$$

and

$$
\operatorname{dimker} M_{\phi}^{*} \cap\left[\mathcal{M}_{0}\left(\psi_{2}\right)\right]^{\perp}=2 .
$$

This is a contradiction. Thus $\left\{\mathcal{M}_{0}(\phi),\left[\mathcal{M}_{0}\left(\psi_{2}\right) \ominus \mathcal{M}_{0}(\phi)\right],\left[\mathcal{M}_{0}\left(\psi_{2}\right)\right]^{\perp}\right\}$ are exact nontrivial minimal reducing subspaces of $M_{\phi}$.

(3) If $\phi$ is not decomposable, by Lemma 2.3, then $\phi$ equals $z^{3} \phi_{\alpha}$ or $z^{2} \phi_{\alpha} \phi_{\beta}$ for two nonzero points $\alpha \beta$ in $\mathbb{D}$ and $\alpha$ does not equal $\beta$ or $-\beta$. The difficult cases will be dealt with in Sections 3 and 4. By Theorems 3.1 and 4.1, $M_{\phi}$ has exact two nontrivial minimal reducing subspaces $\left\{\mathcal{M}_{0}(\phi), \mathcal{M}_{0}(\phi)^{\perp}\right\}$.

\section{REDUCING SUBSPACES OF $M_{z^{3} \phi_{\alpha}}$}

In this section we will study reducing subspaces of $M_{z^{3} \phi_{\alpha}}$ for a nonzero point $\alpha \in \mathbb{D}$. Recall that $\mathcal{M}_{0}$ is the distinguished reducing subspace of $\phi(\mathcal{B})$ as in Theorem 1.2.

Theorem 3.1. Let $\phi=z^{3} \phi_{\alpha}$ for a nonzero point $\alpha \in \mathbb{D}$. Then $\phi(\mathcal{B})$ has exact two nontrivial reducing subspaces $\left\{\mathcal{M}_{0}, \mathcal{M}_{0}^{\perp}\right\}$.

Proof. Let $\mathcal{M}_{0}$ be the distinguished reducing subspace of $\phi(\mathcal{B})$ as in Theorem 1.2. By Theorem 1.3, we only need to show that $\mathcal{M}_{0}^{\perp}$ is a minimal reducing subspace for $\phi(\mathcal{B})$.

Assume that $\mathcal{M}_{0}^{\perp}$ is not a minimal reducing subspace for $\phi(\mathcal{B})$. Then by Theorem 3.1 in [16] we may assume

$$
\mathcal{H}=\bigoplus_{i=0}^{2} M_{i}
$$

such that each $M_{i}$ is a nontrivial reducing subspace for $\phi(\mathcal{B}), M_{0}=\mathcal{M}_{0}$ is the distinguished reducing subspace for $\phi(\mathcal{B})$ and

$$
\mathcal{M}_{0}^{\perp}=M_{1} \oplus M_{2}
$$

Recall that

$$
\begin{gathered}
\phi_{0}=z^{2} \phi_{\alpha}, \\
L_{0}=\operatorname{span}\left\{1, p_{1}, p_{2}, k_{\alpha}(z) k_{\alpha}(w)\right\},
\end{gathered}
$$

and

$$
L_{0}=\left(L_{0} \cap M_{0}\right) \oplus\left(L_{0} \cap M_{1}\right) \oplus\left(L_{0} \cap M_{2}\right) .
$$

We further assume that

$$
\operatorname{dim}\left(M_{1} \cap L_{0}\right)=1
$$

and

$$
\operatorname{dim}\left(M_{2} \cap L_{0}\right)=2 .
$$


Take $0 \neq e_{1} \in M_{1} \cap L_{0}, e_{2}, e_{3} \in M_{2} \cap L_{0}$ such that $\left\{e_{2}, e_{3}\right\}$ are a basis for $M_{2} \cap L_{0}$, then

$$
L_{0}=\operatorname{span}\left\{e_{0}, e_{1}, e_{2}, e_{3}\right\}
$$

By (1.1), we have

$$
d_{e_{j}}^{0}=w e_{j}(0, w) e_{0}-\phi(w) e_{j}
$$

and direct computations show that

$$
\begin{aligned}
\left\langle d_{e_{j}}^{0}, p_{k}\right\rangle & =\left\langle w e_{j}(0, w) e_{0}-\phi(w) e_{j}, p_{k}\right\rangle \\
& =\left\langle w e_{j}(0, w) e_{0}, p_{k}\right\rangle \quad\left(b y T_{\phi(w)}^{*} p_{k}=0\right) \\
& =\left\langle w e_{j}(0, w) e_{0}(w, w), p_{k}(0, w)\right\rangle \\
& =\left\langle w e_{j}(0, w) \phi^{\prime}(w), w^{k}\right\rangle \\
& =\left\langle w^{3} e_{j}(0, w)\left(w \phi_{\alpha}^{\prime}(w)+3 \phi_{\alpha}(w)\right), w^{k}\right\rangle \\
& =\left\langle w^{3-k} e_{j}(0, w)\left(w \phi_{\alpha}^{\prime}(w)+3 \phi_{\alpha}(w)\right), 1\right\rangle \\
& =0
\end{aligned}
$$

for $0 \leq k \leq 2$, and

$$
\begin{aligned}
\left\langle d_{e_{j}}^{0}, k_{\alpha}(z) k_{\alpha}(w)\right\rangle & =\alpha e_{j}(0, \alpha) e_{0}(\alpha, \alpha) \\
& =\alpha e_{j}(0, \alpha) \frac{\alpha^{3}}{1-|\alpha|^{2}} .
\end{aligned}
$$

This implies that those functions $d_{e_{j}}^{0}$ are orthogonal to $\left\{1, p_{1}, p_{2}\right\}$.

Simple calculations give

$$
\left\langle e_{0}, p_{k}\right\rangle=0
$$

for $0 \leq k \leq 1$,

$$
\begin{aligned}
\left\langle e_{0}, p_{2}\right\rangle & =\left\langle e_{0}(0, w), p_{2}(w, w)\right\rangle \\
& =\frac{3}{2} \phi_{0}^{\prime \prime}(0) \\
& =-3 \alpha \neq 0
\end{aligned}
$$

and

$$
\begin{aligned}
\left\langle e_{0}, k_{\alpha}(z) k_{\alpha}(w)\right\rangle & =e_{0}(\alpha, \alpha) \\
& =\phi^{\prime}(\alpha) \\
& =\frac{\alpha^{3}}{1-|\alpha|^{2}} \neq 0
\end{aligned}
$$

By Theorem 1.1, there are numbers $\mu, \lambda_{j}$ such that

$$
\begin{gathered}
d_{e_{1}}^{1}=d_{e_{1}}^{0}+\mu e_{1}+\lambda_{1} e_{0} \\
d_{e_{2}}^{1}=d_{e_{2}}^{0}+\tilde{e_{2}}+\lambda_{2} e_{0} \\
d_{e_{3}}^{1}=d_{e_{3}}^{0}+\tilde{e_{3}}+\lambda_{3} e_{0}
\end{gathered}
$$

where $\tilde{e_{2}}, \tilde{e_{3}} \in M_{2} \cap L_{0}$.

Now we consider two cases. In each case we will derive a contradiction.

Case 1. $\mu \neq 0$. In this case, we get that $e_{1}$ is orthogonal to $\left\{1, p_{1}\right\}$. So $\left\{1, p_{1}, e_{0}, e_{1}\right\}$ form an orthogonal basis for $L_{0}$. 
First we show that $\tilde{e_{2}}=0$. If $\tilde{e_{2}} \neq 0$, then we get that $\left\{1, p_{1}, e_{0}, \tilde{e_{2}}\right\}$ are also an orthogonal basis for $L_{0}$. Thus

$$
\tilde{e_{2}}=c e_{1}
$$

for some nonzero number $c$. However, $\tilde{e_{2}}$ is orthogonal to $e_{1}$ since $\tilde{e_{2}} \in M_{2}$ and $e_{1} \in M_{1}$. This is a contradiction. Thus

$$
d_{e_{2}}^{1}=d_{e_{2}}^{0}+\lambda_{2} e_{0} .
$$

Since both $d_{e_{2}}^{1}$ and $d_{e_{2}}^{0}$ are orthogonal to $p_{2}$ and

$$
\left\langle e_{0}, p_{2}\right\rangle=-3 \alpha \neq 0,
$$

we have that $\lambda_{2}=0$ to get that $d_{e_{2}}^{0}=d_{e_{2}}^{1}$ is orthogonal to $L_{0}$. On the other hand,

$$
\left\langle d_{e_{2}}^{0}, k_{\alpha}(z) k_{\alpha}(w)\right\rangle=\alpha e_{2}(0, \alpha) \frac{\alpha^{3}}{1-|\alpha|^{2}} .
$$

Thus

$$
e_{2}(0, \alpha)=0
$$

Similarly we get that

$$
e_{3}(0, \alpha)=0 .
$$

Moreover, since $e_{2}$ and $e_{3}$ are orthogonal to $\left\{e_{0}, e_{1}\right\}$, write

$$
\begin{aligned}
& e_{2}=c_{11}+c_{12} p_{1}, \\
& e_{3}=c_{21}+c_{22} p_{1} .
\end{aligned}
$$

Thus we have

$$
\begin{aligned}
& e_{2}(0, \alpha)=c_{11}+c_{12} \alpha=0, \\
& e_{3}(0, \alpha)=c_{21}+c_{22} \alpha=0,
\end{aligned}
$$

to get that $e_{2}$ and $e_{3}$ are linearly dependent. This leads to a contradiction in this case.

Case 2. $\mu=0$. In this case we have

$$
d_{e_{1}}^{1}=d_{e_{1}}^{0}+\lambda_{1} e_{0} .
$$

Similarly to the proof in Case 1 we get that $\lambda_{1}=0$,

$$
d_{e_{1}}^{1}=d_{e_{1}}^{0} \perp L_{0}
$$

and

$$
e_{1}(0, \alpha)=0 .
$$

Theorem 2.2 in [16] gives that at least one $\tilde{e}_{j}$, say $\tilde{e}_{2}$ does not equal 0 . Assume that $\tilde{e}_{2} \neq 0$, write

$$
\tilde{e_{2}}=d_{e_{2}}^{1}-d_{e_{2}}^{0}-\lambda_{2} e_{0} \text {. }
$$

Note that we have shown above that both $d_{e_{2}}^{0}$ and $e_{0}$ are orthogonal to both 1 and $p_{1}$. Thus

$$
\tilde{e}_{2} \perp\left\{1, p_{1}\right\}
$$

and

$$
L_{0}=\operatorname{span}\left\{1, p_{1}, e_{0}, \tilde{e}_{2}\right\} .
$$

Since $e_{1}$ is orthogonal to $\left\{e_{0}, \tilde{e}_{2}\right\}$ we have

$$
e_{1}=c_{1}+c_{2} p_{1} .
$$

Noting that $e_{1}(0, \alpha)=c_{1}+c_{2} \alpha=0$ we get

$$
e_{1}=c_{2}\left(-\alpha+p_{1}\right) \text {. }
$$


Without loss of generality we assume that

$$
e_{1}=-\alpha+p_{1}
$$

Letting $e$ be in $M_{2} \cap L_{0}$ such that $e$ is a nonzero function orthogonal to $\tilde{e}_{2}$, we have that $e$ is orthogonal to $\left\{e_{0}, \tilde{e}_{2}\right\}$. Thus $e$ must be in the subspace $\operatorname{span}\left\{1, p_{1}\right\}$. So there are two constants $b_{1}$ and $b_{2}$ such that

$$
e=b_{1}+b_{2} p_{1}
$$

Noting

$$
\begin{aligned}
0 & =\left\langle e, e_{1}\right\rangle \\
& =-b_{1} \bar{\alpha}+2 b_{2}
\end{aligned}
$$

we have

$$
e=\frac{b_{1}}{2}\left(2+\bar{\alpha} p_{1}\right)
$$

Hence we may assume that

$$
e=2+\bar{\alpha} p_{1}
$$

By Theorem 1.1 we have

$$
d_{e}^{1}=d_{e}^{0}+\tilde{e}+\lambda e_{0}
$$

for some number $\lambda$ and $\tilde{e} \in M_{2} \cap L_{0}$. Thus

$$
\begin{aligned}
0 & =\left\langle d_{e_{1}}^{1}, d_{e}^{1}\right\rangle \\
& =\left\langle d_{e_{1}}^{1}, d_{e}^{0}+\tilde{e}+\lambda e_{0}\right\rangle \\
& =\left\langle d_{e_{1}}^{1}, d_{e}^{0}\right\rangle \\
& =\left\langle d_{e_{1}}^{0}, d_{e}^{0}\right\rangle \quad \quad(\text { by }(3.1)) .
\end{aligned}
$$

However, a simple computation gives

$$
\begin{aligned}
\left\langle d_{e_{1}}^{0}, d_{e}^{0}\right\rangle & =\left\langle d_{e_{1}}^{0}, w e(0, w) e_{0}-\phi(w) e\right\rangle \\
& =\left\langle d_{e_{1}}^{0}, w e(0, w) e_{0}\right\rangle \quad\left(b y T_{\phi(w)}^{*} d_{e_{1}}^{0}=0\right) \\
& =\left\langle w e_{1}(0, w) e_{0}-\phi(w) e_{1}, w e(0, w) e_{0}\right\rangle \\
& =\left\langle w e_{1}(0, w) e_{0}, w e(0, w) e_{0}\right\rangle-\left\langle\phi(w) e_{1}, w e(0, w) e_{0}\right\rangle
\end{aligned}
$$

We need to calculate two terms in the right hand of the above equality. By (3.2) and (3.3), the first term becomes

$$
\begin{aligned}
& \left\langle w e_{1}(0, w) e_{0}, w e(0, w) e_{0}\right\rangle \\
= & \left\langle w(-\alpha+w) e_{0}, w(2+\bar{\alpha} w) e_{0}\right\rangle \\
= & \left\langle(-\alpha+w) e_{0},(2+\bar{\alpha} w) e_{0}\right\rangle \\
= & \left\langle-\alpha e_{0}, 2 e_{0}\right\rangle+\left\langle w e_{0}, 2 e_{0}\right\rangle+\left\langle-\alpha e_{0}, \bar{\alpha} w e_{0}\right\rangle+\left\langle w e_{0}, \bar{\alpha} w e_{0}\right\rangle \\
= & -\alpha\left\langle e_{0}, e_{0}\right\rangle+2\left\langle w e_{0}, e_{0}\right\rangle-\alpha^{2}\left\langle e_{0}, w e_{0}\right\rangle .
\end{aligned}
$$


The first term in right hand of the last equality is

$$
\begin{aligned}
\left\langle e_{0}, e_{0}\right\rangle & =\left\langle e_{0}(w, w), e_{0}(0, w)\right\rangle \\
& =\left\langle w \phi_{0}^{\prime}+\phi_{0}, \phi_{0}\right\rangle \\
& =\left\langle w\left(2 w \phi_{\alpha}+w^{2} \phi_{\alpha}^{\prime}\right), w^{2} \phi_{\alpha}\right\rangle+\left\langle\phi_{0}, \phi_{0}\right\rangle . \\
& =2+\left\langle w \phi_{\alpha}^{\prime}, \phi_{\alpha}\right\rangle+1 \\
& =4 .
\end{aligned}
$$

The last equality follows from

$$
\begin{aligned}
\phi_{\alpha} & =-\frac{1}{\bar{\alpha}}+\frac{\frac{1}{\bar{\alpha}}-\alpha}{1-\bar{\alpha} w} \\
& =-\frac{1}{\bar{\alpha}}+\left(\frac{1}{\bar{\alpha}}-\alpha\right) K_{\alpha}(w) .
\end{aligned}
$$

Similarly, we have

$$
\begin{aligned}
\left\langle w e_{0}, e_{0}\right\rangle & =\left\langle w e_{0}(w, w), e_{0}(0, w)\right\rangle \\
& =\left\langle w\left(w \phi_{0}^{\prime}+\phi_{0}\right), \phi_{0}\right\rangle \\
& =\alpha
\end{aligned}
$$

This gives

$$
\begin{aligned}
\left\langle w e_{1}(0, w) e_{0}, w e(0, w) e_{0}\right\rangle= & \left\langle e_{1}(0, w) e_{0}, e(0, w) e_{0}\right\rangle \\
= & \left\langle(-\alpha+w) e_{0},(2+\bar{\alpha} w) e_{0}\right\rangle \\
= & -2 \alpha\left\langle e_{0}, e_{0}\right\rangle-\alpha^{2}\left\langle e_{0}, w e_{0}\right\rangle \\
& +2\left\langle w e_{0}, e_{0}\right\rangle+\alpha\left\langle w e_{0}, w e_{0}\right\rangle \\
= & -8 \alpha-\alpha|\alpha|^{2}+2 \alpha+4 \alpha \\
= & -2 \alpha-\alpha|\alpha|^{2}
\end{aligned}
$$

A simple calculation gives that the second term becomes

$$
\begin{aligned}
& \left\langle\phi(w) e_{1}, w e(0, w) e_{0}\right\rangle \\
= & \left\langle\phi_{0}(w) e_{1},(2+\bar{\alpha} w) e_{0}\right\rangle \\
= & \left\langle\phi_{0}(w) e_{1}, 2 e_{0}\right\rangle+\left\langle\phi_{0}(w) e_{1}, \bar{\alpha} w e_{0}\right\rangle \\
= & 2\left\langle\phi_{0}(w) e_{1}(w, w), e_{0}(0, w)\right\rangle+\alpha\left\langle\phi_{0}(w) e_{1}(w, w), w e_{0}(0, w)\right\rangle \\
= & 2\left\langle e_{1}(w, w), 1\right\rangle+\alpha\left\langle e_{1}(w, w), w\right\rangle \\
= & 2\langle-\alpha+2 w, 1\rangle+\alpha\langle-\alpha+2 w, w\rangle=-2 \alpha+2 \alpha=0 .
\end{aligned}
$$

Thus we conclude

$$
\begin{aligned}
\left\langle d_{e_{1}}^{0}, d_{e}^{0}\right\rangle & =\left\langle w e_{1}(0, w) e_{0}, w e(0, w) e_{0}\right\rangle-\left\langle\phi(w) e_{1}, w e(0, w) e_{0}\right\rangle \\
& =-2 \alpha-\alpha|\alpha|^{2} \\
& =-\alpha\left(2+|\alpha|^{2}\right) \neq 0
\end{aligned}
$$

to get a contradiction in this case. This completes the proof. 


\section{REDUCING SUBSPACES FOR $M_{z^{2} \phi_{\alpha} \phi_{\beta}}$}

In this section we will classify minimal reducing subspaces of $M_{z^{2} \phi_{\alpha} \phi_{\beta}}$ for two nonzero points $\alpha$ and $\beta$ in $\mathbb{D}$ and with $\alpha \neq \beta$.

Theorem 4.1. Let $\phi$ be the Blaschke product $z^{2} \phi_{\alpha} \phi_{\beta}$ for two nonzero points $\alpha$ and $\beta$ in $\mathbb{D}$. If $\alpha$ does not equal either $\beta$ or $-\beta$, then $\phi(\mathcal{B})$ has exact two nontrivial reducing subspaces $\left\{\mathcal{M}_{0}, \mathcal{M}_{0}^{\perp}\right\}$.

Proof. By Theorem 27 in [9], if $\mathcal{N}$ is a nontrivial minimal reducing subspace of $\phi(\mathcal{B})$ which is not equal to $\mathcal{M}_{0}$ then $\mathcal{N}$ is a subspace of $\mathcal{M}_{0}^{\perp}$, so we only need to show that $\mathcal{M}_{0}^{\perp}$ is a minimal reducing subspace for $\phi(\mathcal{B})$ unless $\alpha=-\beta$.

Assume that $\mathcal{M}_{0}^{\perp}$ is not a minimal reducing subspace for $\phi(\mathcal{B})$. By Theorem 3.1 in [16], we may assume

$$
\mathcal{H}=\bigoplus_{i=0}^{2} M_{i}
$$

such that each $M_{i}$ is a reducing subspace for $\phi(\mathcal{B}), M_{0}=\mathcal{M}_{0}$ is the distinguished reducing subspace for $\phi(\mathcal{B})$ and

$$
M_{1} \oplus M_{2}=\mathcal{M}_{0}^{\perp} .
$$

Recall that

$$
\begin{gathered}
\phi_{0}=z \phi_{\alpha} \phi_{\beta}, \\
L_{0}=\operatorname{span}\left\{1, p_{1}, e_{\alpha}, e_{\beta}\right\},
\end{gathered}
$$

with $e_{\alpha}=k_{\alpha}(z) k_{\alpha}(w), e_{\beta}=k_{\beta}(z) k_{\beta}(w)$ and

$$
L_{0}=\left(L_{0} \cap M_{0}\right) \oplus\left(L_{0} \cap M_{1}\right) \oplus\left(L_{0} \cap M_{2}\right) .
$$

So we further assume that the dimension of $M_{1} \cap L_{0}$ is one and the dimension of $M_{2} \cap L_{0}$ is two. Take a nonzero element $e_{1}$ in $M_{1} \cap L_{0}$, then by Theorem 1.1, there are numbers $\mu_{1}, \lambda_{1}$ such that

$$
d_{e_{1}}^{1}=d_{e_{1}}^{0}+\mu_{1} e_{1}+\lambda_{1} e_{0} .
$$

We only need to consider two possibilities, $\mu_{1}$ is zero or nonzero.

If $\mu_{1}$ is zero, then (4.1) becomes

$$
d_{e_{1}}^{1}=d_{e_{1}}^{0}+\lambda_{1} e_{0} .
$$

In this case, simple calculations give

$$
\begin{aligned}
\left\langle d_{e_{1}}^{0}, p_{1}\right\rangle & =\left\langle w e_{1}(0, w) e_{0}(z, w)-w \phi_{0}(w) e_{1}(z, w), p_{1}(z, w)\right\rangle \\
& =\left\langle w e_{1}(0, w) e_{0}(w, w)-w \phi_{0}(w) e_{1}(w, w), p_{1}(z, w)\right\rangle \\
& =\left\langle w e_{1}(0, w) e_{0}(w, w)-w \phi_{0}(w) e_{1}(w, w), p_{1}(0, w)\right\rangle \\
& =\left\langle w e_{1}(0, w) e_{0}(w, w)-w \phi_{0}(w) e_{1}(w, w), w\right\rangle \\
& =\left\langle e_{1}(0, w) e_{0}(w, w)-\phi_{0}(w) e_{1}(w, w), 1\right\rangle \\
& =e_{1}(0,0) e_{0}(0,0)-\phi_{0}(0) e_{1}(0,0)=0,
\end{aligned}
$$


and

$$
\begin{aligned}
\left\langle e_{0}, p_{1}\right\rangle & =\left\langle e_{0}(z, w), p_{1}(z, w)\right\rangle \\
& =\left\langle e_{0}(z, w), p_{1}(w, w)\right\rangle \\
& =\left\langle e_{0}(0, w), 2 w\right\rangle \\
& =\left\langle\phi_{0}(w), 2 w\right\rangle \\
& =2\left\langle w \phi_{\alpha}(w) \phi_{\beta}(w), w\right\rangle \\
& =2 \phi_{\alpha}(0) \phi_{\beta}(0)=2 \alpha \beta \neq 0 .
\end{aligned}
$$

Noting that $d_{e_{1}}^{1}$ is orthogonal to $L_{0}$, by (4.2) we have that $\lambda_{1}=0$, and hence

$$
d_{e_{1}}^{0}=d_{e_{1}}^{1} \perp L_{0} .
$$

So

$$
\left\langle d_{e_{1}}^{0}, e_{\alpha}\right\rangle=0=\left\langle d_{e_{1}}^{0}, e_{\beta}\right\rangle
$$

On the other hand,

$$
\begin{aligned}
\left\langle d_{e_{1}}^{0}, e_{\alpha}\right\rangle & =\alpha e_{1}(0, \alpha) e_{0}(\alpha, \alpha)-\alpha \phi_{0}(\alpha) e_{1}(\alpha, \alpha) \\
& =\alpha e_{1}(0, \alpha) e_{0}(\alpha, \alpha)
\end{aligned}
$$

and

$$
\begin{aligned}
\left\langle d_{e_{1}}^{0}, e_{\beta}\right\rangle & =\beta e_{1}(0, \beta) e_{0}(\beta, \beta)-\beta \phi_{0}(\beta) e_{1}(\beta, \beta) \\
& =\beta e_{1}(0, \beta) e_{0}(\beta, \beta) .
\end{aligned}
$$

Consequently

$$
e_{1}(0, \alpha)=e_{1}(0, \beta)=0 .
$$

Observe that $e_{0}, e_{1}$ and 1 are linearly independent. If this is not so, then $1=a e_{0}+b e_{1}$ for some numbers $a, b$. But $e_{1}(0, \alpha)=0$ and $e_{0}(0, \alpha)=0$. This forces that $1=0$ and leads to a contradiction.

By Theorem 1.1, we can take an element $e \in M_{2} \cap L_{0}$ such that

$$
d_{e}^{1}=d_{e}^{0}+e_{2}+\mu e_{0}
$$

with $e_{2} \neq 0$ and $e_{2} \in M_{2} \cap L_{0}$. Thus we have that $e_{2}$ is orthogonal to 1 and so $e_{2}$ is in $\left\{1, e_{0}, e_{1}\right\}^{\perp}$ and $\left\{1, e_{0}, e_{1}, e_{2}\right\}$ form a basis for $L_{0}$. Moreover for any $f \in M_{2} \cap L_{0}$,

$$
d_{f}^{1}=d_{f}^{0}+g+\lambda e_{0}
$$

for some number $\lambda$ and $g \in M_{2} \cap L_{0}$. If $g$ does not equal 0 then $g$ is orthogonal to 1 . Thus $g$ is in $\left\{1, e_{0}, e_{1}\right\}^{\perp}$ and hence

$$
g=c e_{2}
$$

for some number $c$. Therefore taking a nonzero element $e_{3} \in M_{2} \cap L_{0}$ which is orthogonal to $e_{2}$, we have

$$
\begin{aligned}
& d_{e_{2}}^{1}=d_{e_{2}}^{0}+\mu_{2} e_{2}+\lambda_{2} e_{0}, \\
& d_{e_{3}}^{1}=d_{e_{3}}^{0}+\mu_{3} e_{2}+\lambda_{3} e_{0},
\end{aligned}
$$

and $\left\{e_{0}, e_{1}, e_{2}, e_{3}\right\}$ is an orthogonal basis for $L_{0}$. 
If $\mu_{2}=0$, then by the same reason as before we get

$$
\begin{aligned}
\lambda_{2} & =0, \\
d_{e_{2}}^{0} & =d_{e_{2}}^{1} \perp L_{0} \\
e_{2}(0, \alpha) & =e_{2}(0, \beta) \\
& =0 .
\end{aligned}
$$

So using

$$
p_{1} \in L_{0}=\operatorname{span}\left\{1, e_{0}, e_{1}, e_{2}\right\}
$$

we have

$$
\alpha=p_{1}(0, \alpha)=p_{1}(0, \beta)=\beta,
$$

which contradicts our assumption that $\alpha \neq \beta$. Hence $\mu_{2} \neq 0$.

Observe that 1 is in $L_{0}=\operatorname{span}\left\{e_{0}, e_{1}, e_{2}, e_{3}\right\}$ and orthogonal to both $e_{0}$ and $e_{2}$. Thus

$$
1=c_{1} e_{1}+c_{3} e_{3}
$$

for some numbers $c_{1}$ and $c_{3}$. So

$$
\begin{aligned}
1 & =c_{1} e_{1}(0, \alpha)+c_{3} e_{3}(0, \alpha) \\
& =c_{1} e_{1}(0, \beta)+c_{3} e_{3}(0, \beta) .
\end{aligned}
$$

By (4.3), we have

$$
1=c_{3} e_{3}(0, \alpha)=c_{3} e_{3}(0, \beta),
$$

to obtain that $c_{3} \neq 0$ and

$$
e_{3}(0, \alpha)=e_{3}(0, \beta)=1 / c_{3} .
$$

If $\mu_{3}=0$, then by the same reason as before we get $e_{3}(0, \alpha)=e_{3}(0, \beta)=0$. Hence $\mu_{3} \neq 0$.

Now by the linearality of $d_{(\cdot)}^{1}$ and $d_{(\cdot)}^{0}$ we have

$$
d_{\mu_{3} e_{2}-\mu_{2} e_{3}}^{1}=d_{\mu_{3} e_{2}-\mu_{2} e_{3}}^{0}+\left(\mu_{3} \lambda_{2}-\mu_{2} \lambda_{3}\right) e_{0} .
$$

By the same reason as before we get

$$
\mu_{3} \lambda_{2}-\mu_{2} \lambda_{3}=0
$$

and

and therefore

$$
d_{\mu_{3} e_{2}-\mu_{2} e_{3}}^{0}=d_{\mu_{3} e_{2}-\mu_{2} e_{3}}^{1} \perp L_{0}
$$

$$
\begin{aligned}
\mu_{3} e_{2}(0, \alpha)-\mu_{2} e_{3}(0, \alpha) & =\mu_{3} e_{2}(0, \beta)-\mu_{2} e_{3}(0, \beta) \\
& =0 .
\end{aligned}
$$

So we get

$$
e_{2}(0, \alpha)=\mu_{2} / \mu_{3} c_{3}=e_{2}(0, \beta) .
$$

Hence

$$
p_{1} \in L_{0}=\operatorname{span}\left\{1, e_{0}, e_{1}, e_{2}\right\}
$$

This implies that

$$
\alpha=p_{1}(0, \alpha)=p_{1}(0, \beta)=\beta
$$

which again contradicts our assumption that $\alpha \neq \beta$. 
Another case is that $\mu_{1}$ is not equal to 0 . In this case, (4.1) can be rewritten as

$$
e_{1}=\frac{1}{\mu_{1}} d_{e_{1}}^{1}-\frac{1}{\mu_{1}} d_{e_{1}}^{0}-\frac{\lambda_{1}}{\mu_{1}} e_{0},
$$

and we have that $e_{1}$ is orthogonal to 1 since $d_{e_{1}}^{1}, d_{e_{1}}^{0}$ and $e_{0}$ are orthogonal to 1 . Thus 1 is in $M_{2} \cap L_{0}$.

By Theorem 1.1, there is an element $e \in M_{2} \cap L_{0}$ and a number $\lambda_{0}$ such that

$$
d_{1}^{1}=d_{1}^{0}+e+\lambda_{0} e_{0} .
$$

If $e=0$ then $\lambda_{0}=0$, and hence $d_{1}^{0} \perp L_{0}$ and

$$
1=1(0, \alpha)=1(0, \beta) \text {. }
$$

So $e \neq 0$.

Since $d_{1}^{1}$ is in $L_{0}^{\perp}, d_{1}^{1}$ is orthogonal to 1 . Noting that $d_{1}^{0}$ and $e_{0}$ are orthogonal to 1 , we have that $e \perp 1$. Hence we get an orthogonal basis $\left\{e_{0}, e_{1}, 1, e\right\}$ of $L_{0}$.

Claim.

$$
e(0, \alpha)-e(0, \beta)=0
$$

Proof of the claim. Using Theorem 1.1 again, we have that

$$
d_{e}^{1}=d_{e}^{0}+g+\lambda e_{0}
$$

for some $g \in L_{0} \cap M_{2}$. If $g \neq 0$, we have that $g \perp 1$ since $d_{e}^{1}, d_{e}^{0}$, and $e_{0}$ are orthogonal to 1 . Thus we have that $g=\mu e$ for some number $\mu$ to obtain

$$
d_{e}^{1}=d_{e}^{0}+\mu e+\lambda e_{0} .
$$

Furthermore by the linearality of $d_{(\cdot)}^{1}$ and $d_{(\cdot)}^{0}$ we have that

$$
d_{e-\mu 1}^{1}=d_{e-\mu 1}^{0}+\left(\lambda-\mu \lambda_{0}\right) e_{0} .
$$

By the same reason (namely $d_{e-\mu 1}^{1} \perp L_{0}, d_{e-\mu 1}^{0} \perp 1$ and $\left\langle e_{0}, 1\right\rangle \neq 0$ ) we have that

$$
\begin{gathered}
\lambda-\mu \lambda_{0}=0, \\
d_{e-\mu 1}^{0}=d_{e-\mu 1}^{1} \perp L_{0}
\end{gathered}
$$

and

$$
(e-\mu 1)(0, \alpha)=(e-\mu 1)(0, \beta)=0 .
$$

Hence we have

$$
e(0, \alpha)-e(0, \beta)=\mu-\mu=0,
$$

to complete the proof of the claim.

Let us find the value of $\lambda_{0}$ in (4.4) which will be used to make the coefficients symmetric with respect to $\alpha$ and $\beta$. To do this, we first state a technical lemma which will be used in several other places in the sequel.

Lemma 4.2. If $g$ is in $H^{2}(\mathbb{T})$, then

$$
\left\langle w g \phi_{0}^{\prime}, \phi_{0}\right\rangle=g(0)+g(\alpha)+g(\beta) .
$$


Proof. Since $\phi_{0}$ equals $z \phi_{\alpha} \phi_{\beta}$, simple calculations give

$$
\begin{aligned}
\left\langle w g \phi_{0}^{\prime}, \phi_{0}\right\rangle & =\left\langle w g\left(w \phi_{\alpha} \phi_{\beta}\right)^{\prime}, w \phi_{\alpha} \phi_{\beta}\right\rangle \\
& =\left\langle g\left(w \phi_{\alpha} \phi_{\beta}\right)^{\prime}, \phi_{\alpha} \phi_{\beta}\right\rangle \\
& =\left\langle g\left(\phi_{\alpha} \phi_{\beta}+w \phi_{\alpha}^{\prime} \phi_{\beta}+w \phi_{\alpha} \phi_{\beta}^{\prime}\right), \phi_{\alpha} \phi_{\beta}\right\rangle \\
& =\langle g, 1\rangle+\left\langle w g \phi_{\alpha}^{\prime}, \phi_{\alpha}\right\rangle+\left\langle w g \phi_{\beta}^{\prime}, \phi_{\beta}\right\rangle \\
& =g(0)+\left\langle w g \phi_{\alpha}^{\prime}, \phi_{\alpha}\right\rangle+\left\langle w g \phi_{\beta}^{\prime}, \phi_{\beta}\right\rangle
\end{aligned}
$$

Writing $\phi_{\alpha}$ as

$$
\begin{aligned}
\phi_{\alpha} & =-\frac{1}{\bar{\alpha}}+\frac{\frac{1}{\bar{\alpha}}-\alpha}{1-\bar{\alpha} w} \\
& =-\frac{1}{\bar{\alpha}}+\frac{1-|\alpha|^{2}}{\bar{\alpha}} k_{\alpha}(w),
\end{aligned}
$$

we have

$$
\begin{aligned}
\left\langle w g \phi_{\alpha}^{\prime}, \phi_{\alpha}\right\rangle & =\frac{1-|\alpha|^{2}}{\alpha}\left(w g \phi_{\alpha}^{\prime}\right)(\alpha) \\
& =g(\alpha) .
\end{aligned}
$$

The first equality follows from $\left\langle w g \phi_{\alpha}^{\prime}, 1\right\rangle$ equals 0 and the second equality follows from

$$
\phi_{\alpha}^{\prime}(\alpha)=\frac{1}{1-|\alpha|^{2}}
$$

By the symmetry of $\alpha$ and $\beta$, similar computations lead to

$$
\left\langle w g \phi_{\beta}^{\prime}, \phi_{\beta}\right\rangle=g(\beta)
$$

and the proof is finished.

We state the values of $\lambda_{0}$ and $\left\langle e_{0}, e_{0}\right\rangle$ as a lemma.

\section{Lemma 4.3.}

$$
\begin{gathered}
\lambda_{0}=-\frac{\alpha+\beta}{4} \\
\left\langle e_{0}, e_{0}\right\rangle=4
\end{gathered}
$$

Proof. Since $d_{1}^{1}$ is orthogonal to $L_{0}, e_{0}$ is in $L_{0}$, and $e$ is orthogonal to $e_{0}$, (4.4) gives

$$
\begin{aligned}
0 & =\left\langle d_{1}^{1}, e_{0}\right\rangle \\
& =\left\langle d_{1}^{0}+e+\lambda_{0} e_{0}, e_{0}\right\rangle \\
& =\left\langle d_{1}^{0}, e_{0}\right\rangle+\lambda_{0}\left\langle e_{0}, e_{0}\right\rangle .
\end{aligned}
$$


We need to compute $\left\langle d_{1}^{0}, e_{0}\right\rangle$ and $\left\langle e_{0}, e_{0}\right\rangle$ respectively.

$$
\begin{aligned}
\left\langle d_{1}^{0}, e_{0}\right\rangle & =\left\langle-\phi(w)+w e_{0}, e_{0}\right\rangle \\
& =\left\langle w e_{0}, e_{0}\right\rangle \\
& =\left\langle w e_{0}(w, w), e_{0}(0, w)\right\rangle \\
& =\left\langle w\left(w \phi_{0}^{\prime}+\phi_{0}\right), \phi_{0}\right\rangle \\
& =\left\langle w^{2} \phi_{0}^{\prime}, \phi_{0}\right\rangle+\left\langle w \phi_{0}, \phi_{0}\right\rangle \\
& =\left\langle w^{2} \phi_{0}^{\prime}, \phi_{0}\right\rangle \\
& =\alpha+\beta .
\end{aligned}
$$

The last equality follows from Lemma 4.2 with $g=w$.

$$
\begin{aligned}
\left\langle e_{0}, e_{0}\right\rangle & =\left\langle e_{0}(w, w), e_{0}(0, w)\right\rangle \\
& =\left\langle w \phi_{0}^{\prime}+\phi_{0}, \phi_{0}\right\rangle \\
& =\left\langle w \phi_{0}^{\prime}, \phi_{0}\right\rangle+\left\langle\phi_{0}, \phi_{0}\right\rangle \\
& =\left\langle w \phi_{0}^{\prime}, \phi_{0}\right\rangle+1 \\
& =4,
\end{aligned}
$$

where the last equality follows from Lemma 4.2 with $g=1$. Hence

$$
\alpha+\beta+4 \lambda_{0}=0
$$

and

$$
\lambda_{0}=-\frac{\alpha+\beta}{4} .
$$

Let $P_{L_{0}}$ denote the projection of $H^{2}\left(\mathbb{T}^{2}\right)$ onto $L_{0}$. The element $P_{L_{0}}\left(k_{\alpha}(w)-k_{\beta}(w)\right)$ has the property that for any $g \in L_{0}$,

$$
\begin{aligned}
\left\langle g, P_{L_{0}}\left(k_{\alpha}(w)-k_{\beta}(w)\right)\right\rangle & =\left\langle g, k_{\alpha}(w)-k_{\beta}(w)\right\rangle \\
& =g(0, \alpha)-g(0, \beta) .
\end{aligned}
$$

Thus $P_{L_{0}}\left(k_{\alpha}(w)-k_{\beta}(w)\right)$ is orthogonal to $g$ for $g \in L_{0}$ with

$$
g(0, \alpha)=g(0, \beta) .
$$

So $P_{L_{0}}\left(k_{\alpha}(w)-k_{\beta}(w)\right)$ is orthogonal to $e_{0}, 1, e$. On the other hand,

$$
\begin{aligned}
\left\langle p_{1}, P_{L_{0}}\left(k_{\alpha}(w)-k_{\beta}(w)\right)\right\rangle & =\alpha-\beta \\
& \neq 0 .
\end{aligned}
$$

This gives that the element $P_{L_{0}}\left(k_{\alpha}(w)-k_{\beta}(w)\right)$ is a nonzero element. Therefore there exists a nonzero number $b$ such that

$$
P_{L_{0}}\left(k_{\alpha}(w)-k_{\beta}(w)\right)=b e_{1} .
$$

Without loss of generality we assume that

$$
e_{1}=P_{L_{0}}\left(k_{\alpha}(w)-k_{\beta}(w)\right) .
$$


Observe that

$$
\begin{aligned}
p_{1}(\phi(z), \phi(w)) e_{1}+d_{e_{1}}^{1} & \in M_{1}, \\
p_{1}(\phi(z), \phi(w))+d_{1}^{1} & \in M_{2}, \\
M_{1} & \perp M_{2},
\end{aligned}
$$

to get

$$
\left\langle p_{1}(\phi(z), \phi(w)) e_{1}+d_{e_{1}}^{1}, p_{1}(\phi(z), \phi(w))+d_{1}^{1}\right\rangle=0
$$

Thus we have

$$
\begin{aligned}
0 & =\left\langle p_{1}(\phi(z), \phi(w)) e_{1}+d_{e_{1}}^{1}, p_{1}(\phi(z), \phi(w))+d_{1}^{1}\right\rangle \\
& =\left\langle(\phi(z)+\phi(w)) e_{1}, \phi(z)+\phi(w)\right\rangle+\left\langle d_{e_{1}}^{1}, d_{1}^{1}\right\rangle \\
& =\left\langle d_{e_{1}}^{1}, d_{1}^{1}\right\rangle
\end{aligned}
$$

The second equality follows from

$$
d_{e_{1}}^{1}, d_{1}^{1} \in \operatorname{ker} T_{\phi(z)}^{*} \cap \operatorname{ker} T_{\phi(z)}^{*} .
$$

The last equality follows from

$$
e_{1} \perp 1
$$

and

$$
e_{1}, 1 \in \operatorname{ker} T_{\phi(z)}^{*} \cap \operatorname{ker} T_{\phi(z)}^{*} .
$$

Substituting (4.4) into Equation (4.7), we have

$$
\begin{aligned}
0 & =\left\langle d_{e_{1}}^{1}, d_{1}^{0}+e+\lambda_{0} e_{0}\right\rangle \\
& =\left\langle d_{e_{1}}^{1}, d_{1}^{0}\right\rangle \\
& =\left\langle d_{e_{1}}^{1},-\phi(w)+w e_{0}\right\rangle \\
& =\left\langle d_{e_{1}}^{1}, w e_{0}\right\rangle \\
& =\left\langle d_{e_{1}}^{0}+\mu_{1} e_{1}+\lambda_{1} e_{0}, w e_{0}\right\rangle \\
& =\left\langle d_{e_{1}}^{0}, w e_{0}\right\rangle+\mu_{1}\left\langle e_{1}, w e_{0}\right\rangle+\lambda_{1}\left\langle e_{0}, w e_{0}\right\rangle .
\end{aligned}
$$

The second equation comes from that $d_{e_{1}}^{1}$ is orthogonal to $L_{0}$ and both $e$ and $e_{0}$ are in $L_{0}$. The third equation follows from the definition of $d_{1}^{0}$ and the forth equation follows from that $d_{e_{1}}^{1}$ is in $\operatorname{ker} T_{\phi(z)}^{*} \cap \operatorname{ker} T_{\phi(w)}^{*}$. We need to calculate $\left\langle d_{e_{1}}^{0}, w e_{0}\right\rangle,\left\langle e_{1}, w e_{0}\right\rangle$, and $\left\langle e_{0}, w e_{0}\right\rangle$ separately.

To get $\left\langle d_{e_{1}}^{0}, w e_{0}\right\rangle$, by the definition of $d_{e_{1}}^{0}$, we have

$$
\begin{aligned}
\left\langle d_{e_{1}}^{0}, w e_{0}\right\rangle & =\left\langle-\phi(w) e_{1}+w e_{1}(0, w) e_{0}, w e_{0}\right\rangle \\
& =\left\langle-\phi(w) e_{1}, w e_{0}\right\rangle+\left\langle w e_{1}(0, w) e_{0}, w e_{0}\right\rangle
\end{aligned}
$$

Thus we need to compute $\left\langle-\phi(w) e_{1}, w e_{0}\right\rangle$ and $\left\langle w e_{1}(0, w) e_{0}, w e_{0}\right\rangle$ one by one. The equality

$$
\left\langle-\phi(w) e_{1}, w e_{0}\right\rangle=0
$$


follows from the following computations.

$$
\begin{aligned}
\left\langle-\phi(w) e_{1}, w e_{0}\right\rangle & =\left\langle-w \phi_{0}(w) e_{1}, w e_{0}\right\rangle \\
& =-\left\langle\phi_{0}(w) e_{1}, e_{0}\right\rangle \\
& =-\left\langle\phi_{0}(w) e_{1}(w, w), e_{0}(0, w)\right\rangle \\
& =-\left\langle\phi_{0}(w) e_{1}(w, w), \phi_{0}(w)\right\rangle \\
& =-\left\langle e_{1}(w, w), 1\right\rangle \\
& =-\left\langle e_{1}, 1\right\rangle \\
& =0 .
\end{aligned}
$$

To get $\left\langle w e_{1}(0, w) e_{0}, w e_{0}\right\rangle$, we continue as follows.

$$
\begin{aligned}
\left\langle w e_{1}(0, w) e_{0}, w e_{0}\right\rangle & =\left\langle e_{1}(0, w) e_{0}, e_{0}\right\rangle \\
& =\left\langle e_{1}(0, w) e_{0}(w, w), e_{0}(0, w)\right\rangle \\
& =\left\langle e_{1}(0, w) e_{0}(w, w), \phi_{0}(w)\right\rangle \\
& =\left\langle e_{1}(0, w)\left(\phi_{0}(w)+w \phi_{0}^{\prime}(w)\right), \phi_{0}(w)\right\rangle \\
& =\left\langle e_{1}(0, w) \phi_{0}(w), \phi_{0}(w)\right\rangle+\left\langle e_{1}(0, w) w \phi_{0}^{\prime}(w), \phi_{0}(w)\right\rangle \\
& =\left\langle e_{1}(0, w), 1\right\rangle+\left\langle e_{1}(0, w) w \phi_{0}^{\prime}(w), \phi_{0}(w)\right\rangle \\
& =e_{1}(0,0)+\left\langle e_{1}(0, w) w \phi_{0}^{\prime}(w), \phi_{0}(w)\right\rangle \\
& =\left\langle e_{1}, 1\right\rangle+\left\langle e_{1}(0, w) w \phi_{0}^{\prime}(w), \phi_{0}(w)\right\rangle \\
& =\left\langle e_{1}(0, w) w \phi_{0}^{\prime}(w), \phi_{0}(w)\right\rangle \\
& =e_{1}(0, \alpha)+e_{1}(0, \beta) .
\end{aligned}
$$

The last equality follows from Lemma 4.2 and

$$
e_{1}(0,0)=\left\langle e_{1}, 1\right\rangle=0
$$

Hence

$$
\left\langle d_{e_{1}}^{0}, w e_{0}\right\rangle=e_{1}(0, \alpha)+e_{1}(0, \beta)
$$

Recall that

$$
d_{1}^{1}=d_{1}^{0}+e+\lambda_{0} e_{0}
$$

is orthogonal to $L_{0}$ and $e_{1}$ is orthogonal to both $e$, and $e_{0}$. Thus

$$
\begin{aligned}
0 & =\left\langle e_{1}, d_{1}^{0}+e+\lambda_{0} e_{0}\right\rangle \\
& =\left\langle e_{1},-\phi(w)+w e_{0}\right\rangle \\
& =\left\langle e_{1}, w e_{0}\right\rangle .
\end{aligned}
$$

From the computation of $\left\langle d_{1}^{0}, e_{0}\right\rangle$ in the proof of Lemma 4.3 we have showed that

$$
\left\langle w e_{0}, e_{0}\right\rangle=\alpha+\beta .
$$

Therefore we have that

$$
e_{1}(0, \alpha)+e_{1}(0, \beta)+\lambda_{1}(\bar{\alpha}+\bar{\beta})=0 .
$$


On the other hand,

$$
\begin{aligned}
0 & =\left\langle d_{e_{1}}^{1}, e_{0}\right\rangle \\
& =\left\langle d_{e_{1}}^{0}+\mu_{1} e_{1}+\lambda_{1} e_{0}, e_{0}\right\rangle \\
& =\left\langle d_{e_{1}}^{0}, e_{0}\right\rangle+4 \lambda_{1}
\end{aligned}
$$

and

$$
\begin{aligned}
\left\langle d_{e_{1}}^{0}, e_{0}\right\rangle & =\left\langle-\phi(w) e_{1}+w e_{1}(0, w) e_{0}, e_{0}\right\rangle \\
& =\left\langle w e_{1}(0, w) e_{0}, e_{0}\right\rangle \\
& =\left\langle w e_{1}(0, w) e_{0}(w, w), e_{0}(0, w)\right\rangle \\
& =\left\langle w e_{1}(0, w)\left(\phi_{0}(w)+w \phi_{0}^{\prime}\right), \phi_{0}(w)\right\rangle \\
& =\left\langle w^{2} e_{1}(0, w) \phi_{0}^{\prime}, \phi_{0}(w)\right\rangle \\
& =\alpha e_{1}(0, \alpha)+\beta e_{1}(0, \beta) .
\end{aligned}
$$

The last equality follows from Lemma 4.2 with $g=w e_{1}(0, w)$. Thus

$$
\alpha e_{1}(0, \alpha)+\beta e_{1}(0, \beta)+4 \lambda_{1}=0 .
$$

So

$$
\lambda_{1}=-\frac{\alpha}{4} e_{1}(0, \alpha)-\frac{\beta}{4} e_{1}(0, \beta) .
$$

Substituting (4.9) into (4.8), we have

$$
\left[1-\frac{\alpha(\bar{\alpha}+\bar{\beta})}{4}\right] e_{1}(0, \alpha)+\left[1-\frac{\beta(\bar{\alpha}+\bar{\beta})}{4}\right] e_{1}(0, \beta)=0 .
$$

Recall that

$$
\lambda_{0}=-\frac{\alpha+\beta}{4}
$$

to get

$$
\left(1+\bar{\lambda}_{0} \alpha\right) e_{1}(0, \alpha)+\left(1+\bar{\lambda}_{0} \beta\right) e_{1}(0, \beta)=0 .
$$

We are going to draw another equation about $e_{1}(0, \alpha)$ and $e_{1}(0, \beta)$ from the property that $d_{e_{1}}^{1}$ is orthogonal to $L_{0}$. To do this, recall that

$$
\begin{aligned}
e_{1} & =P_{L_{0}}\left(k_{\alpha}(w)-k_{\beta}(w)\right) \in M_{1} \cap L_{0}, \\
d_{e_{1}}^{1} & =d_{e_{1}}^{0}+\mu_{1} e_{1}+\lambda_{1} e_{0} \perp L_{0}, \\
L_{0} & =\operatorname{span}\left\{1, p_{1}, e_{\alpha}, e_{\beta}\right\} \\
e_{\alpha} & =k_{\alpha}(z) k_{\alpha}(w), e_{\beta}=k_{\beta}(z) k_{\beta}(w) .
\end{aligned}
$$

Thus $d_{e_{1}}^{1}$ is orthogonal to $p_{1}, e_{\alpha}$ and $e_{\beta}$.

Since $d_{e_{1}}^{1}$ is orthogonal to $p_{1}$ we have

$$
\left\langle d_{e_{1}}^{0}, p_{1}\right\rangle+\mu_{1}\left\langle e_{1}, p_{1}\right\rangle+\lambda_{1}\left\langle e_{0}, p_{1}\right\rangle=0 .
$$


Noting

$$
\begin{aligned}
\left\langle d_{e_{1}}^{0}, p_{1}\right\rangle & =\left\langle-\phi(w) e_{1}+w e_{1}(0, w) e_{0}, p_{1}\right\rangle \\
& =\left\langle w e_{1}(0, w) e_{0}, p_{1}\right\rangle \\
& =\left\langle w e_{1}(0, w) e_{0}(w, w), w\right\rangle \\
& =\left\langle e_{1}(0, w) e_{0}(w, w), 1\right\rangle \\
& =0 \\
\left\langle e_{1}, p_{1}\right\rangle & =\left\langle P_{L_{0}}\left(K_{\alpha}(w)-K_{\beta}(w)\right), p_{1}\right\rangle \\
& =\left\langle K_{\alpha}(w)-K_{\beta}(w), p_{1}\right\rangle \\
& =\bar{\alpha}-\bar{\beta}
\end{aligned}
$$

and

$$
\begin{aligned}
\left\langle e_{0}, p_{1}\right\rangle & =\left\langle e_{0}(0, w), p_{1}(w, w)\right\rangle \\
& =\left\langle\phi_{0}(w), 2 w\right\rangle \\
& =\left\langle w \phi_{\alpha} \phi_{\beta}, 2 w\right\rangle \\
& =2\left\langle\phi_{\alpha} \phi_{\beta}, 1\right\rangle \\
& =2 \phi_{\alpha}(0) \phi_{\beta}(0) \\
& =2 \alpha \beta
\end{aligned}
$$

we have

$$
(\bar{\alpha}-\bar{\beta}) \mu_{1}+2 \alpha \beta \lambda_{1}=0
$$

to obtain

$$
\lambda_{1}=-\mu_{1} \frac{\bar{\alpha}-\bar{\beta}}{2 \alpha \beta}
$$

Since $d_{e_{1}}^{1} \perp e_{\alpha}$, we have

$$
\left\langle d_{e_{1}}^{0}, e_{\alpha}\right\rangle+\mu_{1}\left\langle e_{1}, e_{\alpha}\right\rangle+\lambda_{1}\left\langle e_{0}, e_{\alpha}\right\rangle=0
$$

to get

$$
\left\langle d_{e_{1}}^{0}, e_{\alpha}\right\rangle+\mu_{1}\left\langle e_{1}, e_{\alpha}\right\rangle-\mu_{1} \frac{\bar{\alpha}-\bar{\beta}}{2 \alpha \beta}\left\langle e_{0}, e_{\alpha}\right\rangle=0 .
$$

We need to calculate $\left\langle d_{e_{1}}^{0}, e_{\alpha}\right\rangle,\left\langle e_{1}, e_{\alpha}\right\rangle$ and $\left\langle e_{0}, e_{\alpha}\right\rangle$. Simple calculations show that

$$
\begin{aligned}
\left\langle d_{e_{1}}^{0}, e_{\alpha}\right\rangle & =\left\langle-\phi(w) e_{1}+w e_{1}(0, w) e_{0}, e_{\alpha}\right\rangle \\
& =\left\langle w e_{1}(0, w) e_{0}, e_{\alpha}\right\rangle \\
& =\alpha e_{1}(0, \alpha) e_{0}(\alpha, \alpha) \\
\left\langle e_{1}, e_{\alpha}\right\rangle & =e_{1}(\alpha, \alpha) \\
& =\left\langle P_{L_{0}}\left(k_{\alpha}(w)-k_{\beta}(w)\right), e_{\alpha}\right\rangle \\
& =\left\langle k_{\alpha}(w)-k_{\beta}(w), e_{\alpha}\right\rangle \\
& =\frac{1}{1-|\alpha|^{2}}-\frac{1}{1-\alpha \bar{\beta}}
\end{aligned}
$$




$$
=\frac{\alpha(\bar{\alpha}-\bar{\beta})}{\left(1-|\alpha|^{2}\right)(1-\alpha \bar{\beta})},
$$

and

$$
\begin{aligned}
\left\langle e_{0}, e_{\alpha}\right\rangle= & e_{0}(\alpha, \alpha)=\alpha \phi_{0}^{\prime}(\alpha)+\phi_{0}(\alpha) \\
& =\alpha^{2} \frac{1}{1-|\alpha|^{2}} \frac{\alpha-\beta}{1-\alpha \bar{\beta}}
\end{aligned}
$$

Thus (4.13) and (4.14) give

$$
\frac{e_{1}(\alpha, \alpha)}{e_{0}(\alpha, \alpha)}=\frac{\bar{\alpha}-\bar{\beta}}{\alpha(\alpha-\beta)} .
$$

Substituting the above equality in Equation (4.12) leads to

$$
\alpha e_{1}(0, \alpha) e_{0}(\alpha, \alpha)+\mu_{1} e_{1}(\alpha, \alpha)-\mu_{1} \frac{\bar{\alpha}-\bar{\beta}}{2 \alpha \beta} e_{0}(\alpha, \alpha)=0 .
$$

Dividing the both sides of the above equality by $e_{0}(\alpha, \alpha)$ gives

$$
\alpha e_{1}(0, \alpha)+\mu_{1} \frac{e_{1}(\alpha, \alpha)}{e_{0}(\alpha, \alpha)}-\mu_{1} \frac{\bar{\alpha}-\bar{\beta}}{2 \alpha \beta}=0 .
$$

Hence we have

$$
\alpha e_{1}(0, \alpha)+\mu_{1} \frac{\bar{\alpha}-\bar{\beta}}{\alpha(\alpha-\beta)}-\mu_{1} \frac{\bar{\alpha}-\bar{\beta}}{2 \alpha \beta}=0
$$

to obtain

$$
\alpha e_{1}(0, \alpha)+\left(\beta+\lambda_{0}\right) \frac{2 \mu_{1}(\bar{\alpha}-\bar{\beta})}{\alpha \beta(\alpha-\beta)}=0 .
$$

Similarly, since $d_{e_{1}}^{1}$ is orthogonal to $e_{\beta}$, we have

$$
\left\langle d_{e_{1}}^{0}, e_{\beta}\right\rangle+\mu_{1}\left\langle e_{1}, e_{\beta}\right\rangle+\lambda_{1}\left\langle e_{0}, e_{\beta}\right\rangle=0,
$$

to obtain

$$
\left\langle d_{e_{1}}^{0}, e_{\beta}\right\rangle+\mu_{1}\left\langle e_{1}, e_{\beta}\right\rangle-\mu_{1} \frac{\bar{\alpha}-\bar{\beta}}{2 \alpha \beta}\left\langle e_{0}, e_{\beta}\right\rangle=0 .
$$

We need to calculate $\left\langle d_{e_{1}}^{0}, e_{\beta}\right\rangle,\left\langle e_{1}, e_{\beta}\right\rangle$ and $\left\langle e_{0}, e_{\beta}\right\rangle$. Simple calculations as above show that

$$
\begin{aligned}
\left\langle d_{e_{1}}^{0}, e_{\beta}\right\rangle & =\left\langle-\phi(w) e_{1}+w e_{1}(0, w) e_{0}, e_{\beta}\right\rangle \\
& =\left\langle w e_{1}(0, w) e_{0}, e_{\beta}\right\rangle \\
& =\beta e_{1}(0, \beta) e_{0}(\beta, \beta) \\
\left\langle e_{1}, e_{\beta}\right\rangle & =e_{1}(\beta, \beta) \\
& =\left\langle P_{L_{0}}\left(k_{\alpha}(w)-k_{\beta}(w)\right), e_{\beta}\right\rangle \\
& =\left\langle k_{\alpha}(w)-k_{\beta}(w), e_{\beta}\right\rangle \\
& =\frac{1}{1-\bar{\alpha} \beta}-\frac{1}{1-|\beta|^{2}}
\end{aligned}
$$




$$
\begin{aligned}
& =\frac{\beta(\bar{\alpha}-\bar{\beta})}{(1-\bar{\alpha} \beta)\left(1-|\beta|^{2}\right)} \\
\left\langle e_{0}, e_{\beta}\right\rangle= & e_{0}(\beta, \beta)=\beta \phi_{0}^{\prime}(\beta)+\phi_{0}(\beta) \\
& =\beta^{2} \frac{\beta-\alpha}{1-\bar{\alpha} \beta} \frac{1}{1-|\beta|^{2}}
\end{aligned}
$$

Combining (4.17) with (4.18) gives

$$
\frac{e_{1}(\beta, \beta)}{e_{0}(\beta, \beta)}=-\frac{\bar{\alpha}-\bar{\beta}}{\beta(\alpha-\beta)} .
$$

Substituting the above equality in (4.16) gives

$$
\beta e_{1}(0, \beta) e_{0}(\beta, \beta)+\mu_{1} e_{1}(\beta, \beta)-\mu_{1} \frac{\bar{\alpha}-\bar{\beta}}{2 \alpha \beta} e_{0}(\beta, \beta)=0 .
$$

Dividing both sides of the above equality by $e_{0}(\beta, \beta)$ gives

$$
\beta e_{1}(0, \beta)+\mu_{1} \frac{e_{1}(\beta, \beta)}{e_{0}(\beta, \beta)}-\mu_{1} \frac{\bar{\alpha}-\bar{\beta}}{2 \alpha \beta}=0
$$

Hence we have

$$
\beta e_{1}(0, \beta)-\mu_{1} \frac{\bar{\alpha}-\bar{\beta}}{\beta(\alpha-\beta)}-\mu_{1} \frac{\bar{\alpha}-\bar{\beta}}{2 \alpha \beta}=0
$$

to get

$$
\beta e_{1}(0, \beta)-\left(\alpha+\lambda_{0}\right) \frac{2 \mu_{1}(\bar{\alpha}-\bar{\beta})}{\alpha \beta(\alpha-\beta)}=0 .
$$

Eliminating $\frac{2 \mu_{1}(\bar{\alpha}-\bar{\beta})}{\alpha \beta(\alpha-\beta)}$ from (4.15) and (4.19) gives

$$
\alpha\left(\alpha+\lambda_{0}\right) e_{1}(0, \alpha)+\beta\left(\beta+\lambda_{0}\right) e_{1}(0, \beta)=0 .
$$

Now combining (4.10) and (4.20), we have the following linear system of equations about $e_{1}(0, \alpha)$ and $e_{1}(0, \beta)$

$$
\begin{gathered}
\left(1+\bar{\lambda}_{0} \alpha\right) e_{1}(0, \alpha)+\left(1+\bar{\lambda}_{0} \beta\right) e_{1}(0, \beta)=0 \\
\alpha\left(\alpha+\lambda_{0}\right) e_{1}(0, \alpha)+\beta\left(\beta+\lambda_{0}\right) e_{1}(0, \beta)=0 .
\end{gathered}
$$

If

$$
e_{1}(0, \alpha)=e_{1}(0, \beta)=0,
$$

then $p_{1}$ is in $L_{0}=\operatorname{span}\left\{e_{0}, e_{1}, 1, e\right\}$. But noting

$$
e_{0}(0, \alpha)=e_{0}(0, \beta)
$$

and

$$
e(0, \alpha)=e(0, \beta)
$$

we have

$$
p_{1}(0, \alpha)=p_{1}(0, \beta),
$$


which contradicts the assumption that $\alpha \neq \beta$. So at least one of $e_{1}(0, \alpha)$ and $e_{1}(0, \beta)$ is nonzero. Then the determinant of the coefficient matrix of System (4.21) has to be zero. This implies

$$
\left|\begin{array}{cc}
1+\bar{\lambda}_{0} \alpha & 1+\bar{\lambda}_{0} \beta \\
\alpha\left(\alpha+\lambda_{0}\right) & \beta\left(\beta+\lambda_{0}\right)
\end{array}\right|=0
$$

Making elementary row reductions on the above the determinant, we get

$$
\left|\begin{array}{cc}
(\alpha-\beta) \bar{\lambda}_{0} & 1+\bar{\lambda}_{0} \beta \\
(\alpha-\beta)\left(\alpha+\beta+\lambda_{0}\right) & \beta\left(\beta+\lambda_{0}\right)
\end{array}\right|=0 .
$$

Since

$$
\alpha+\beta=-4 \lambda_{0}
$$

and

we have

$$
\alpha-\beta \neq 0
$$

$$
\left|\begin{array}{cc}
\bar{\lambda}_{0} & 1+\bar{\lambda}_{0} \beta \\
-3 \lambda_{0} & \beta\left(\beta+\lambda_{0}\right)
\end{array}\right|=0 .
$$

Expanding this determinant we have

$$
\begin{aligned}
0 & =\bar{\lambda}_{0}\left(\beta^{2}+\beta \lambda_{0}\right)+3 \lambda_{0}\left(1+\bar{\lambda}_{0} \beta\right) \\
& =\bar{\lambda}_{0}\left(\beta^{2}+\beta \lambda_{0}+3 \beta \lambda_{0}\right)+3 \lambda_{0} \\
& =\bar{\lambda}_{0}\left(\beta^{2}+4 \beta \lambda_{0}\right)+3 \lambda_{0} \\
& =\bar{\lambda}_{0}(-\alpha \beta)+3 \lambda_{0}
\end{aligned}
$$

Taking absolute value on both sides of the above equation, we have

$$
\begin{aligned}
0 & =\left|\bar{\lambda}_{0}(-\alpha \beta)+3 \lambda_{0}\right| \\
& \geq\left|\lambda_{0}\right|(3-|\alpha \beta|) \\
& \geq 2\left|\lambda_{0}\right|
\end{aligned}
$$

to get

$$
\lambda_{0}=0
$$

This implies

$$
\alpha+\beta=0
$$

to complete the proof.

\section{REFERENCES}

[1] I. Baker, J. Deddens and J. Ullman, A theorem on entire functions with applications to Toeplitz operators, Duke Math. J. 41(1974), 739-745.

[2] J. Ball, Hardy space expectation operators and reducing subspaces, Proc. Amer. Math. Soc. 47(1975), 351-357.

[3] H. Bercovici, C. Foias and C. Pearcy, Dual algebra with applications to invariant subspace and dilation theory, CBMS, Vol. 56.

[4] A. Brown, On a class of operators, Proc. Amer. Math. Soc. 4 (1953) 723-728.

[5] R. Douglas and V. Paulsen, Hilbert Modules over function algebras, Pitman Research Notes in Mathematics, Series 217, Lonman Group UK Limited, 1989.

[6] R. Douglas, R. Yang, Operator theory in the Hardy space over the bidisk. I. Integral Equations Operator Theory 38 (2000), 207-221. 
[7] S. Ferguson and R. Rochberg, Higher order Hilbert-Schmidt Hankel forms and tensors of analytic kernels, Math. Scand., 96 (2005), no. 1, 117-146.

[8] S. Ferguson and R. Rochberg, Description of certain quotient Hilbert modules, Operator theory, 20, 93-109, Theta Ser. Adv. Math., 6, Theta, Bucharest, 2006.

[9] K. Guo, S. Sun, D. Zheng and C. Zhong, Multiplication Operators on the Bergman Space via the Hardy Space of the bidisk, to appear in J. Reine Angew. Math.

[10] P. Halmos, Shifts on Hilbert spaces, J. Reine. Angew. Math. 208(1961) 102-112.

[11] E. Nordgren, Reducing subspaces of analytic Toeplitz operators, Duke Math. J. 34(1967), 175-181.

[12] S. Richter, On invariant subspaces of multiplication operators on Banach spaces of analytic functions, $\mathrm{Ph}$. Dissertation, Univ. of Michigan, 1986.

[13] K. Stephenson, Analytic functions of finite valence, with applications to Toeplitz operators, Michigan Math. J, 32 (1985), 5-19.

[14] M. Stessin and K. Zhu, Reducing subspace of weighted shift operators, Proc. Amer. Math. Soc., 130 (2002), 2631-2639.

[15] M. Stessin and K. Zhu, Generalized factorization in Hardy spaces and the commutant of Toeplitz operators. Canad. J. Math. 55 (2003), no. 2, 379-400.

[16] S. Sun, D. Zheng and C. Zhong, Multiplication Operators on the Bergman Space and Weighted shifts, to appear in J. Operator theory

[17] J. Walsh, On the location of the roots of the jacobian of two binary forms, and of the derivative of a rational function, Trans. Amer. Math. Soc. 19(1918), 291-298.

[18] R. Yang, Operator theory in the Hardy space over the bidisk. II. Integral Equations Operator Theory 42 (2002), 99-124.

[19] R. Yang, Operator theory in the Hardy space over the bidisk. III. J. Funct. Anal. 186 (2001), 521-545.

[20] R. Yang, The core operator and congruent submodules. J. Funct. Anal. 228 (2005), 469-489.

[21] K. Zhu, Reducing subspaces for a class of multiplication operators, J. London Math. Soc., (2) 62(2000), 553-568.

Institute of Mathematics, Jiaxing University, JiAXing, ZheJiang, 314001, P. R. ChinA

E-mail address: shsun@mail.zjxu.edu.cn

Department of Mathematics, VANDERbilt University, NAShVille, TN 37240 USA

E-mail address: dechao.zhengevanderbilt.edu

Department of Mathematics \& Statistics, Georgia State University Atlanta, Georgia 30303, USA

E-mail address: matcyz@langate.gsu . edu 